\title{
Stability results for obstacle problems with measure data
}

\section{Résultats de stabilité pour des problèmes d'obstacle avec données mesure}

\author{
Chiara Leone \\ Dipartimento di Matematica e Applicazioni “R. Caccioppoli”, Università “Federico II” di Napoli, Via Cintia, 80126 Napoli, Italy \\ Received 3 January 2000; received in revised form 1 October 2002; accepted 9 March 2005
}

Available online 22 June 2005

\begin{abstract}
We study the convergence properties of the solutions of some elliptic obstacle problems with measure data, under the simultaneous perturbation of the operator, the forcing term and the obstacle.
\end{abstract}

(C) 2005 L'Association Publications de l'Institut Henri Poincaré. Published by Elsevier B.V. All rights reserved

\section{Résumé}

On étudie les propriétés de convergence des solutions de problémes d'obstacles elliptiques avec données mesures, lorsque l'opérateur differentiel, les données ou les obstacles changent.

(C) 2005 L'Association Publications de l'Institut Henri Poincaré. Published by Elsevier B.V. All rights reserved

MSC: 35J85; 35R05; 49K40

\section{Introduction}

Obstacle problems when the data do not belong to the dual of the energy space have been considered in $[6,5,13$, 19,20], where the authors studied the notion of solution of a unilateral problem for a monotone operator $A(u)=$ $-\operatorname{div}(a(x, \nabla u))$ acting on $W_{0}^{1, p}(\Omega), p>1$, when the forcing term is a bounded Radon measure $\mu$ vanishing on all sets of $p$-capacity zero (see Section 1 for the definition of $p$-capacity).

The problem we deal in this paper regards the behaviour of the obstacle problem with measure data under perturbation of the operator, of the forcing term, and of the obstacle.

E-mail address: chileone@unina.it (C. Leone). 
We begin with some remarks on the obstacle problem in the variational framework. For any datum $F \in$ $W^{-1, p^{\prime}}(\Omega)$ and for any function $\psi: \Omega \rightarrow \widetilde{\mathbb{R}}$, the unilateral problem relative to $A, F$, and the obstacle $\psi$ (denoted by $\operatorname{VI}(A, F, \psi))$ is the problem of finding a function $u$ such that

$$
\left\{\begin{array}{l}
u \in W_{0}^{1, p}(\Omega), \quad u \geqslant \psi, \\
\langle A(u), v-u\rangle \geqslant\langle F, v-u\rangle, \\
\forall v \in W_{0}^{1, p}(\Omega), \quad v \geqslant \psi .
\end{array}\right.
$$

This problem has a unique solution whenever the convex set

$$
K_{\psi}:=\left\{z \in W_{0}^{1, p}(\Omega): z \geqslant \psi C_{p} \text {-q.e. in } \Omega\right\}
$$

is nonempty.

In [16] (see also [8]) the authors proved some results on the convergence of variational inequalities for monotone operators, when both the operator and the obstacle are perturbed. They considered a sequence of variational inequalities $\operatorname{VI}\left(A_{h}, F_{h}, \psi_{h}\right)$ and the corresponding convex sets

$$
K_{\psi_{h}}:=\left\{z \in W_{0}^{1, p}(\Omega): z \geqslant \psi_{h} C_{p} \text {-q.e. in } \Omega\right\},
$$

assuming that

$$
\begin{aligned}
& F_{h} \text { converges to } F \text { strongly in } W_{0}^{1, p}(\Omega), \\
& a_{h} G \text {-converges to } a, \\
& K_{\psi_{h}} \text { converges to } K_{\psi} \text { in the sense of Mosco. }
\end{aligned}
$$

Denoting the solutions of $\operatorname{VI}\left(A_{h}, F_{h}, \psi_{h}\right)$ and $\operatorname{VI}(A, F, \psi)$ by $u_{h}$ and $u$, respectively, Theorem 3.1 of [16] shows that

$$
\begin{aligned}
& u_{h} \rightarrow u \quad \text { weakly in } W_{0}^{1, p}(\Omega), \\
& a_{h}\left(x, \nabla u_{h}\right) \rightarrow a(x, \nabla u) \quad \text { weakly in } L^{p^{\prime}}(\Omega)^{N}, \\
& \int_{\Omega} a_{h}\left(x, \nabla u_{h}\right) \nabla u_{h} \mathrm{~d} x \rightarrow \int_{\Omega} a(x, \nabla u) \nabla u \mathrm{~d} x .
\end{aligned}
$$

In this paper we extend the stability result stated above to the case when the forcing term $\mu$ is a bounded Radon measure which vanishes on all sets of $p$-capacity zero, that is to say $\mu \in \mathcal{M}_{b, 0}^{p}(\Omega)$.

We point out that, if the forcing term $\mu \in \mathcal{M}_{b, 0}^{p}(\Omega)$, the classical definition (1.1) given above fails. In this paper we will adopt the notion of solution considered in [22] to solve uniquely the obstacle problem (denoted by $O P(A, \mu, \psi))$, when the forcing term $\mu$ is a measure in $\mathcal{M}_{b, 0}^{p}(\Omega)$.

We consider a sequence of obstacle problems $O P\left(A_{h}, \mu_{h}, \psi_{h}\right)$, when the measures $\mu_{h}$ vanish on sets of $p$-capacity zero, and we assume that

$$
\begin{aligned}
& \mu_{h}(B) \rightarrow \mu(B), \quad \text { for every Borel set } B \subseteq \Omega, \\
& a_{h} G \text {-converges to } a, \\
& K_{\psi_{h}} \text { converge to } K_{\psi} \text { in the sense of Mosco. }
\end{aligned}
$$

Denoting the solutions of $O P\left(A_{h}, \mu_{h}, \psi_{h}\right)$ and $O P(A, \mu, \psi)$ by $u_{h}$ and $u$, respectively, we will prove in Theorem 6.1 that 


$$
\begin{aligned}
& T_{j}\left(u_{h}\right) \rightarrow T_{j}(u) \quad \text { weakly in } W_{0}^{1, p}(\Omega), \quad \text { for every } j>0, \\
& a_{h}\left(x, \nabla u_{h}\right) \rightarrow a(x, \nabla u) \quad \text { weakly in } L^{q}(\Omega)^{N}, \text { for every } q<\frac{N}{N-1}, \\
& \int_{\Omega} a_{h}\left(x, \nabla u_{h}\right) \nabla T_{j}\left(u_{h}\right) \mathrm{d} x \rightarrow \int_{\Omega} a(x, \nabla u) \nabla T_{j}(u) \mathrm{d} x, \quad \text { for every } j>0,
\end{aligned}
$$

where $T_{j}(\cdot)$ is the truncation function at level $j$ (see Section 1 for the definition).

In the special case where $a_{h}=a$, for every $h$, we obtain also that $T_{j}\left(u_{h}\right)$ converges to $T_{j}(u)$ strongly in $W_{0}^{1, p}(\Omega)$, for every $j>0$.

Other results in the case $a_{h}=a$, under different hypotheses on $\mu_{h}$ and $\psi_{h}$, can be found in [12].

\section{Assumptions and notations}

Let $\Omega$ be a bounded, open subset of $\mathbb{R}^{N}, N \geqslant 2$. Let $p$ be a real constant, $1<p \leqslant N$, and let $p^{\prime}$ its dual exponent, $1 / p+1 / p^{\prime}=1$.

Given two constants $c_{0}, c_{1}>0$ and two constants $\alpha$ and $\beta$, with $0 \leqslant \alpha \leqslant 1 \wedge(p-1)$ and $p \vee 2 \leqslant \beta<+\infty$, we consider the family $\mathcal{L}\left(c_{0}, c_{1}, \alpha, \beta\right)$ of Carathéodory functions $a(x, \xi): \Omega \times \mathbb{R}^{N} \rightarrow \mathbb{R}^{N}$ such that:

$$
\begin{aligned}
& |a(x, \xi)-a(x, \eta)| \leqslant c_{0}(1+|\xi|+|\eta|)^{p-1-\alpha}|\xi-\eta|^{\alpha}, \\
& (a(x, \xi)-a(x, \eta))(\xi-\eta) \geqslant c_{1}(1+|\xi|+|\eta|)^{p-\beta}|\xi-\eta|^{\beta}, \\
& a(x, 0)=0,
\end{aligned}
$$

for almost every $x \in \Omega$, for every $\xi, \eta \in \mathbb{R}^{N}$.

Under the assumptions (2.1)-(2.3), the operator $A: u \mapsto-\operatorname{div}(a(x, \nabla u))$ maps $W_{0}^{1, p}(\Omega)$ into its dual $W^{-1, p^{\prime}}(\Omega)$, and for any $F$ in $W^{-1, p^{\prime}}(\Omega)$ there exists a unique solution $u \in W_{0}^{1, p}(\Omega)$ of the equation

$$
\begin{cases}A(u)=F & \text { in } \Omega, \\ u=0 & \text { on } \partial \Omega,\end{cases}
$$

since, in particular, $A$ is coercive, continuous, bounded and strictly monotone (see [22]).

Remark 2.1. For a particular choice of the constants $\alpha$ and $\beta$, i.e. if $1<p \leqslant 2, \alpha=p-1$, and $\beta=2$, the inequalities (2.1) and (2.2) become

$$
\begin{aligned}
& |a(x, \xi)-a(x, \eta)| \leqslant c_{0}|\xi-\eta|^{p-1}, \\
& (a(x, \xi)-a(x, \eta))(\xi-\eta) \geqslant c_{1}(1+|\xi|+|\eta|)^{p-2}|\xi-\eta|^{2} .
\end{aligned}
$$

Moreover, if $2 \leqslant p<+\infty, \alpha=1$, and $\beta=p$, the continuity and monotonicity assumptions (2.1) and (2.2) for the function $a$ take the form

$$
\begin{aligned}
& |a(x, \xi)-a(x, \eta)| \leqslant c_{0}(1+|\xi|+|\eta|)^{p-2}|\xi-\eta|, \\
& (a(x, \xi)-a(x, \eta))(\xi-\eta) \geqslant c_{1}|\xi-\eta|^{p} .
\end{aligned}
$$

We recall that, given a compact set $K \subseteq \Omega$, its $p$-capacity with respect to $\Omega$ is given by

$$
C_{p}(K)=\inf \left\{\int_{\Omega}|\nabla z|^{2} \mathrm{~d} x: z \in C_{0}^{\infty}(\Omega), z \geqslant \chi_{K}\right\},
$$


where $\chi_{K}$ is the characteristic function of $K$. This definition can be extended to any open subset $B$ of $\Omega$ in the following way:

$$
C_{p}(B)=\sup \left\{C_{p}(K): K \text { compact, } K \subseteq B\right\} .
$$

Finally, it is possible to define the $p$-capacity of any set $A \subseteq \Omega$ as:

$$
C_{p}(A)=\inf \left\{C_{p}(B): B \text { open, } A \subseteq B\right\} .
$$

A property holds $C_{p}$-quasi everywhere (abbreviated as $C_{p}$-q.e.) when it holds up to sets of $p$-capacity zero.

A function $v: \Omega \rightarrow \overline{\mathbb{R}}$ is $C_{p}$-quasi Borel if there exists a Borel function $u: \Omega \rightarrow \overline{\mathbb{R}}$ such that $v=u C_{p}$-q.e. in $\Omega$. A function $v: \Omega \rightarrow \overline{\mathbb{R}}$ is $C_{p}$-quasi continuous (resp. $C_{p}$-quasi upper semicontinuous) if, for every $\varepsilon>0$ there exists a set $E$ such that $C_{p}(E)<\varepsilon$ and $v_{\mid \Omega \backslash E}$ is continuous (resp. upper semicontinuous) in $\Omega \backslash E$. Thus, every $C_{p}$-quasi continuous (resp. $C_{p}$-quasi upper semicontinuous) $v$ is a $C_{p}$-quasi Borel function.

A function $u \in W_{0}^{1, p}(\Omega)$ always has a $C_{p}$-quasi continuous representative, which is uniquely defined (and finite) up to a set of $p$-capacity zero. In the sequel we shall always identify $u$ with its $C_{p}$-quasi continuous representative, so that the pointwise values of $u$ are defined $C_{p}$-quasi everywhere.

A set $E \subseteq \Omega$ is said to be $C_{p}$-quasi open if for every $\varepsilon>0$ there exists an open set $U$ such that $E \subseteq U \subseteq \Omega$ and $C_{p}(U \backslash E) \leqslant \varepsilon$.

Let $\mathcal{M}_{b}(\Omega)$ the space of Radon measures $\mu$ on $\Omega$ whose total variation $|\mu|$ is bounded on $\Omega$, while $\mathcal{M}_{b, 0}^{p}(\Omega)$ is the special subspace of $\mathcal{M}_{b}(\Omega)$ of all measures, which are absolutely continuous with respect to the $p$-capacity, that is a measure $\mu \in \mathcal{M}_{b}(\Omega)$ belongs to $\mathcal{M}_{b, 0}^{p}(\Omega)$ if and only if $\mu(A)=0$ for every Borel set $A \subseteq \Omega$ such that $C_{p}(A)=0$. As usual, we identify $\mathcal{M}_{b}(\Omega)$ with the dual of the Banach space $C_{0}(\Omega)$ of continuous functions that are zero on the boundary; so that the duality is $\langle\mu, u\rangle=\int_{\Omega} u \mathrm{~d} \mu$, for every $u$ in $C_{0}(\Omega)$ and the norm is $\|\mu\|_{\mathcal{M}_{b}(\Omega)}=$ $|\mu|(\Omega)$. Moreover, we denote the positive cones of $\mathcal{M}_{b}(\Omega)$ and $\mathcal{M}_{b, 0}^{p}(\Omega)$ by $\mathcal{M}_{b}^{+}(\Omega)$ and $\mathcal{M}_{b, 0}^{p,+}(\Omega)$, respectively.

It is well known that, if $\mu$ belongs to $W^{-1, p^{\prime}}(\Omega) \cap \mathcal{M}_{b}(\Omega)$, then $\mu$ is in $\mathcal{M}_{b, 0}^{p}(\Omega)$, every $u$ in $W_{0}^{1, p}(\Omega) \cap$ $L^{\infty}(\Omega)$ is summable with respect to $\mu$ and

$$
\langle\mu, u\rangle=\int_{\Omega} u \mathrm{~d} \mu,
$$

where $\langle\cdot, \cdot\rangle$ denotes the duality pairing between $W^{-1, p^{\prime}}(\Omega)$ and $W_{0}^{1, p}(\Omega)$, while in the right-hand side $u$ denotes the $C_{p}$-quasi continuous representative and, consequently, the pointwise values of $u$ are defined $\mu$-almost everywhere.

For every $j>0$ we define the truncation function $T_{j}: \mathbb{R} \mapsto \mathbb{R}$ by

$$
T_{j}(t)= \begin{cases}t & \text { if }|t| \leqslant j \\ j \operatorname{sign}(t) & \text { if }|t|>j\end{cases}
$$

Let us consider the space $\mathcal{T}_{0}^{1, p}(\Omega)$ of all functions $u: \Omega \mapsto \overline{\mathbb{R}}$ which are almost everywhere finite and such that $T_{j}(u) \in W_{0}^{1, p}(\Omega)$ for every $j>0$. It is easy to see that every function $u \in \mathcal{T}_{0}^{1, p}(\Omega)$ has a $C_{p}$-quasi continuous representative with values in $\overline{\mathbb{R}}$, that will always be identified with the function $u$. Moreover, for every $u \in \mathcal{T}_{0}^{1, p}(\Omega)$ there exists a measurable function $\Phi: \Omega \mapsto \mathbb{R}^{N}$ such that $\nabla T_{j}(u)=\Phi \chi_{\{|u| \leqslant j\}}$ a.e. in $\Omega$ (see Lemma 2.1 in [3]). This function $\Phi$, which is unique up to almost everywhere equivalence, will be denoted by $\nabla u$. Note that $\nabla u$ coincides with the distributional gradient of $u$ whenever

$$
u \in \mathcal{T}_{0}^{1, p}(\Omega) \cap L_{\mathrm{loc}}^{1}(\Omega) \text { and } \nabla u \in L_{\mathrm{loc}}^{1}\left(\Omega, \mathbb{R}^{N}\right) .
$$




\section{Entropy solutions and obstacle problems}

We are now in position to recall the notion of entropy solution introduced in [3] for $L^{1}$ data and extended to measures in $\mathcal{M}_{b, 0}^{p}(\Omega)$ in [7], which ensures us that, when $\mu \in \mathcal{M}_{b, 0}^{p}(\Omega)$, the equation

$$
\begin{cases}A(u)=\mu & \text { in } \Omega \\ u=0 & \text { on } \partial \Omega\end{cases}
$$

has a unique entropy solution.

We point out that the theory of entropy solutions works for general Carathéodory functions $a: \Omega \times \mathbb{R}^{N} \rightarrow \mathbb{R}^{N}$ such that, for almost every $x \in \Omega$ and for all $\xi, \eta \in \mathbb{R}^{N}$, with $\xi \neq \eta$,

$$
\begin{aligned}
& |a(x, \xi)| \leqslant c_{2}\left[k(x)+|\xi|^{p-1}\right], \\
& a(x, \xi) \cdot \xi \geqslant c_{3}|\xi|^{p}-g(x), \\
& (a(x, \xi)-a(x, \eta))(\xi-\eta)>0, \\
& a(x, 0)=0,
\end{aligned}
$$

where $c_{2}$ and $c_{3}$ are two positive real constants, $g$ is a nonnegative function in $L^{1}(\Omega)$ and $k$ is a nonnegative function in $L^{p^{\prime}}(\Omega)$ (see Remark 2.4 of [19]).

We note that, if $a \in \mathcal{L}\left(c_{0}, c_{1}, \alpha, \beta\right)$, these conditions are satisfied, with $g$ and $k$ replaced by positive real constants depending on $c_{0}, c_{1}, \alpha$, and $\beta$.

Definition 3.1. Let $\mu \in \mathcal{M}_{b, 0}^{p}(\Omega)$. A function $u$ is an entropy solution of problem (3.1) if $u$ belongs to $\mathcal{T}_{0}^{1, p}(\Omega)$, and

$$
\int_{\Omega} a(x, \nabla u) \nabla T_{j}(u-\varphi) \mathrm{d} x \leqslant \int_{\Omega} T_{j}(u-\varphi) \mathrm{d} \mu,
$$

for every $\varphi$ in $W_{0}^{1, p}(\Omega) \cap L^{\infty}(\Omega)$ and every $j>0$.

Remark 3.2. If $F \in W^{-1, p^{\prime}}(\Omega)$ we can consider as data also $\mu+F$, the definition of entropy solution being

$$
\int_{\Omega} a(x, \nabla u) \nabla T_{j}(u-\varphi) \mathrm{d} x \leqslant \int_{\Omega} T_{j}(u-\varphi) \mathrm{d} \mu+\left\langle F, T_{j}(u-\varphi)\right\rangle .
$$

Remark 3.3. Actually, it is possible to prove that equality holds in (3.6) and (3.7) (see [21]).

Remark 3.4. Using $\varphi=0$ in (3.7), by (3.3) and by Young's inequality, we easily get

$$
\int_{\Omega}\left|\nabla T_{j}(u)\right|^{p} \leqslant c(j+1), \quad \forall j>0
$$

where the constant $c$ depends on $\|\mu\|_{\mathcal{M}_{b}(\Omega)},\|F\|_{W^{-1, p^{\prime}(\Omega)}}, p, c_{3}$, and $\|g\|_{L^{1}(\Omega)}$.

By standard arguments of capacity theory, (3.8) implies

$$
C_{p}(\{|u|>j\}) \leqslant \frac{c(j+1)}{j^{p}}
$$

that is, if $u$ is the entropy solution of (3.1) relative to $\mu+F$, then (the $C_{p}$-quasi continuous representative of) $u$ is finite up to a set of capacity zero. 
Now, using $\varphi=T_{i}(u)$ in (3.7), by (3.9) we get

$$
\lim _{i \rightarrow+\infty} \int_{\{i<|u| \leqslant i+j\}}|\nabla u|^{p} \mathrm{~d} x=0 .
$$

Remark 3.5. By means of (3.8), we can apply Lemma 4.2 of [3] which implies that, for every $1<q<\frac{N}{N-1}$, $|\nabla u|^{p-1}$ is bounded in $L^{q}(\Omega)$ by some constant depending only by $N$ and $c$. Moreover, the procedure used in [7] to obtain the entropy formulation (3.7), combined with the uniqueness of $u$, allows to prove that, for every $\Phi \in W_{0}^{1, q^{\prime}}(\Omega)$, with $1<q<\frac{N}{N-1}$,

$$
\int_{\Omega} a(x, \nabla u) \nabla \Phi \mathrm{d} x=\int_{\Omega} \Phi \mathrm{d} \mu+\langle F, \Phi\rangle,
$$

as well as

$$
\int_{\Omega} a(x, \nabla u) \nabla\left(T_{j}(u-\varphi) \phi\right) \mathrm{d} x=\int_{\Omega} T_{j}(u-\varphi) \phi \mathrm{d} \mu+\left\langle F, T_{j}(u-\varphi) \phi\right\rangle,
$$

for every $\varphi \in W_{0}^{1, p}(\Omega) \cap \mathrm{L}^{\infty}(\Omega)$ and for every $\phi \in C^{1}(\bar{\Omega})$. Let us observe that if $q<\frac{N}{N-1}$, then $q^{\prime}>N$, so that, by Sobolev embedding theorems, $W_{0}^{1, q^{\prime}}(\Omega) \subseteq C(\bar{\Omega})$.

We recall also the following stability result (see Theorem 1.2 in [21] and Remark 3.3 in [19]):

Theorem 3.6. Let $\mu_{h} \in \mathcal{M}_{b, 0}^{p}(\Omega)$ and $F_{h} \in W^{-1, p^{\prime}}(\Omega)$ be such that

$$
\begin{array}{ll}
\mu_{h} \rightarrow \mu & \text { strongly in } \mathcal{M}_{b}(\Omega), \\
F_{h} \rightarrow F & \text { strongly in } W^{-1, p^{\prime}}(\Omega) ;
\end{array}
$$

let $u_{h}$ be the entropy solutions of (3.1) relative to $\mu_{h}+F_{h}$, and let $u$ be the entropy solution of (3.1) relative to $\mu+F$. Then

$$
\lim _{h \rightarrow \infty} T_{j}\left(u_{h}\right)=T_{j}(u) \quad \text { strongly in } W_{0}^{1, p}(\Omega),
$$

for every $j>0$.

Before specifying the notion of solution we will adopt in this paper in order to study obstacle problems when the forcing term is a measure, we want to mention here these two facts, concerning the solution $u$ of $V I(A, F, \psi)$, when $F \in W^{-1, p^{\prime}}(\Omega)$.

Characterization 1. The solution $u$ can be characterized (see, e.g., Chapters II and III in [18]) as the smallest function in $W_{0}^{1, p}(\Omega)$, greater than or equal to $\psi$, such that

$$
\begin{cases}A(u)-F=\lambda & \text { in } \Omega, \\ u=0 & \text { on } \partial \Omega,\end{cases}
$$

for some nonnegative element $\lambda$ of $W^{-1, p^{\prime}}(\Omega)$.

Characterization 2. Finally, when the obstacle $\psi$ is $C_{p}$-quasi upper semicontinuous $u$, is also characterized (see, e.g., Theorem 3.2 in [1]) by the complementarity system

$$
\left\{\begin{array}{l}
u \in W_{0}^{1, p}(\Omega), \quad u \geqslant \psi, \\
A(u)=F+\lambda, \\
\lambda \in W^{-1, p^{\prime}}(\Omega), \quad \lambda \geqslant 0, \\
\lambda(\{u-\psi>0\})=0,
\end{array}\right.
$$


where the pointwise values of $u$ are defined $C_{p}$-quasi everywhere. Since $\lambda$ is a nonnegative element of $W^{-1, p^{\prime}}(\Omega)$, by the Riesz Representation Theorem, it is a nonnegative Radon measure; this explains the meaning of the last line of (3.16), which can be written also as $u=\psi \lambda$-almost everywhere in $\Omega$.

Let us observe that without loss of generality we may suppose that $\psi$ is $C_{p}$-quasi upper semicontinuous thanks to the following proposition (see Proposition 1.5 in [15]).

Proposition 3.7. Let $\psi: \Omega \mapsto \overline{\mathbb{R}}$, with $K_{\psi}$ nonempty. Then there exists a $C_{p}$-quasi upper semicontinuous function $\hat{\psi}: \Omega \mapsto \overline{\mathbb{R}}$ such that:

(1) $\hat{\psi} \geqslant \psi C_{p}$-q.e. in $\Omega$;

(2) if $\varphi: \Omega \mapsto \overline{\mathbb{R}}$ is $C_{p}$-quasi upper semicontinuous and $\varphi \geqslant \psi C_{p}$-q.e. in $\Omega$, then $\varphi \geqslant \hat{\psi} C_{p}$-q.e. in $\Omega$.

Thus, in particular, $K_{\psi}=K_{\hat{\psi}}$.

Besides, let us observe that if $p$ is greater than the dimension $N$ of the ambient space, then it is easily seen, by Sobolev embedding and duality arguments, that the space $\mathcal{M}_{b}(\Omega)$ is a subset of $W^{-1, p^{\prime}}(\Omega)$, so that existence, uniqueness, and continuous dependence of solutions in $W_{0}^{1, p}(\Omega)$ to the obstacle problem was studied as part of the theory of the variational inequality (1.1).

In [19] the following definition for unilateral problems with measure data was introduced.

Definition 3.8. We say that $u$ is the solution of the Obstacle Problem with datum $\mu \in \mathcal{M}_{b, 0}^{p}(\Omega)$ and obstacle $\psi$ if

(1) there exists a measure $\lambda \in \mathcal{M}_{b, 0}^{p,+}(\Omega)$ such that $u$ is the entropy solution of

$$
\begin{cases}A(u)=\mu+\lambda & \text { in } \Omega, \\ u=0 & \text { on } \partial \Omega,\end{cases}
$$

and $u \geqslant \psi C_{p}$-quasi everywhere in $\Omega$.

(2) for any $v \in \mathcal{M}_{b, 0}^{p,+}(\Omega)$ such that the entropy solution $v$ of (3.17) relative to $\mu+v$ satisfies $v \geqslant \psi C_{p}$-quasi everywhere in $\Omega$, we have $u \leqslant v C_{p}$-q.e. in $\Omega$.

By definition, it is clear that, if such a solution exists, it is unique.

The nonnegative measure $\lambda$, which is uniquely defined, will be called the obstacle reaction relative to $u$, or the measure associated with it.

The only restriction required on the choice of the obstacle is that there exists a measure $\rho \in \mathcal{M}_{b}(\Omega) \cap$ $W^{-1, p^{\prime}}(\Omega)$ such that the solution $u_{\rho}$ of

$$
\begin{cases}A\left(u_{\rho}\right)=\rho & \text { in } \Omega, \\ u_{\rho}=0 & \text { on } \partial \Omega\end{cases}
$$

is such that

$$
\psi \leqslant u_{\rho} \quad C_{p} \text {-q.e. in } \Omega .
$$

The following theorem was proved in [19].

Theorem 3.9. Let $\psi$ satisfy (3.18) and let $\mu \in \mathcal{M}_{b, 0}^{p}(\Omega)$. Then there exists a unique solution of $O P(A, \mu, \psi)$. Moreover the corresponding obstacle reaction $\lambda$ satisfies

$$
\|\lambda\|_{\mathcal{M}_{b}(\Omega)} \leqslant\left\|(\mu-\rho)^{-}\right\|_{\mathcal{M}_{b}(\Omega)} .
$$

The solution found can be characterized by the complementarity system. 
Theorem 3.10. Let $\mu$ be in $\mathcal{M}_{b, 0}^{p}(\Omega)$ and $\psi$ satisfy (3.18); then the following statements are equivalent:

(1) $u$ is the solution of $\mathrm{OP}(A, \mu, \psi)$ and $\lambda$ is the associated obstacle reaction;

(2) $u \geqslant \psi C_{p}$-q.e. in $\Omega, \lambda \in \mathcal{M}_{b, 0}^{p,+}(\Omega)$, $u$ is the entropy solution of (3.17) relative to $\mu+\lambda$, and

$$
\left\{\begin{array}{l}
\int_{\Omega} T_{k}(u-\varphi) \mathrm{d} \lambda \leqslant \int_{\Omega} T_{k}(v-\varphi) \mathrm{d} \lambda, \\
\forall \varphi \in W_{0}^{1, p}(\Omega) \cap L^{\infty}(\Omega), \\
\forall v \in \mathcal{T}_{0}^{1, p}(\Omega), v \geqslant \psi \quad C_{p} \text {-q.e. in } \Omega ;
\end{array}\right.
$$

(3) $u \geqslant \psi C_{p}$-q.e. in $\Omega, \lambda \in \mathcal{M}_{b, 0}^{p,+}(\Omega)$, $u$ is the entropy solution of (3.17) relative to $\mu+\lambda$, and

$$
u=\psi \quad \lambda \text {-a.e. in } \Omega \text {. }
$$

Remark 3.11. Observe that if $\psi$ is $C_{p}$-q.e. upper bounded, we can consider in (3.20) $\varphi \in W_{0}^{1, p}(\Omega) \cap L^{\infty}(\Omega)$, $\varphi \geqslant \psi C_{p}$-q.e. in $\Omega$ and $v=\varphi$, so that, taking into account that $u$ is the entropy solution of (3.17) relative to $\mu+\lambda$, $u$ satisfies

$$
\int_{\Omega} a(x, \nabla u) \nabla T_{k}(u-\varphi) \mathrm{d} x \leqslant \int_{\Omega} T_{k}(u-\varphi) \mathrm{d} \mu,
$$

which is quite similar to the usual variational formulation. Formula (3.22) was just obtained in [5] when the datum $\mu$ is a function in $L^{1}(\Omega)$. In that paper L. Boccardo and G.R. Cirmi proved also that formulation (3.22) characterizes uniquely the function $u$. In the same way this can be done also when $\mu \in \mathcal{M}_{b, 0}^{p}(\Omega)$.

\section{4. $G$-convergence, Mosco-convergence, and weak convergence in $\mathcal{M}_{b}(\Omega)$}

The study of the properties of the solutions to the obstacle problems under perturbations of the operator $a$ is based on a notion of convergence in $\mathcal{L}\left(c_{0}, c_{1}, \alpha, \beta\right)$, called $G$-convergence.

Definition 4.1. We say that a sequence of functions $a_{h}(x, \xi)$ belonging to $\mathcal{L}\left(c_{0}, c_{1}, \alpha, \beta\right) G$-converges to a function $a(x, \xi)$ satisfying the same hypotheses (possibly with different constants $\left.\tilde{c}_{0}, \tilde{c}_{1}, \tilde{\alpha}, \tilde{\beta}\right)$ if for any $F \in W^{-1, p^{\prime}}(\Omega)$, the solution $u_{h}$ of

$$
\begin{cases}A_{h}\left(u_{h}\right)=F & \text { in } \Omega, \\ u=0 & \text { on } \partial \Omega\end{cases}
$$

satisfies

$$
u_{h} \rightarrow u \quad \text { weakly in } W_{0}^{1, p}(\Omega)
$$

and

$$
a_{h}\left(x, \nabla u_{h}\right) \rightarrow a(x, \nabla u) \quad \text { weakly in } L^{p^{\prime}}(\Omega)^{N},
$$

where $u$ is the unique solution of (2.4).

The following theorem justifies the definition of $G$-convergence.

Theorem 4.2. Any sequence $a_{h}(x, \xi)$ of functions belonging to $\mathcal{L}\left(c_{0}, c_{1}, \alpha, \beta\right)$ admits a subsequence which $G$-converges to a function $a(x, \xi) \in \mathcal{L}\left(\tilde{c}_{0}, \tilde{c}_{1}, \frac{\alpha}{\beta-\alpha}, \beta\right)$, where $\tilde{c}_{0}, \tilde{c}_{1}$ depend only on $N, p, \alpha, \beta, c_{0}, c_{1}$. 
This compactness theorem was obtained by L. Tartar (see [24] and Theorem 1.1 of [17]) in the case of nonlinear monotone operators defined from $\mathrm{H}_{0}^{1}(\Omega)$ into $\mathrm{H}^{-1}(\Omega)$, when $p=2$ and the functions $a_{h} \in \mathcal{L}\left(c_{0}, c_{1}, 1,2\right)$, and then extended in the version of Theorem 4.2 in [11] (see Theorem 4.1).

The investigations of the properties of obstacle problems when the obstacle varies relies on a notion of convergence for sequences of convex sets introduced by U. Mosco in [23].

Definition 4.3. Let $K_{h}$ be a sequence of subsets of a Banach space $X$. The strong lower limit

$$
s-\liminf _{h \rightarrow+\infty} K_{h}
$$

of the sequence $K_{h}$ is the set of all $v \in X$ such that there exists a sequence $v_{h} \in K_{h}$, for $h$ large, converging to $v$ strongly in $X$.

The weak upper limit

$$
w-\limsup _{h \rightarrow+\infty} K_{h}
$$

of the sequence $K_{h}$ is the set of all $v \in X$ such that there exists a sequence $v_{k}$ converging to $v$ weakly in $X$ and a sequence of integers $h_{k}$ converging to $+\infty$, such that $v_{k} \in K_{h_{k}}$.

The sequence $K_{h}$ converges to the set $K$ in the sense of Mosco, shortly $K_{h} \stackrel{M}{\rightarrow} K$, if

$$
s-\liminf _{h \rightarrow+\infty} K_{h}=w-\limsup _{h \rightarrow+\infty} K_{h}=K .
$$

Mosco proved that this type of convergence is the right one for the stability of variational inequalities with respect to obstacles. This is the main theorem of his theory.

Theorem 4.4. Let $K_{\psi_{h}}$ and $K_{\psi}$ be nonempty. Then

$$
K_{\psi_{h}} \stackrel{M}{\longrightarrow} K_{\psi}
$$

if and only if, for any $F \in W^{-1, p^{\prime}}(\Omega)$,

$$
u_{h} \rightarrow u \text { strongly in } W_{0}^{1, p}(\Omega),
$$

where $u_{h}$ and $u$ are the solutions of $\operatorname{VI}\left(A, F, \psi_{h}\right)$ and $\operatorname{VI}(A, F, \psi)$, respectively.

Several stability results can be proved as corollaries of this theorem by Mosco. In particular, the strong convergence

$$
\psi_{h} \rightarrow \psi \quad \text { strongly in } W_{0}^{1, p}(\Omega)
$$

easily implies the convergence of $K_{\psi_{h}}$ to $K_{\psi}$ in the sense of Mosco, but the weak convergence

$$
\psi_{h} \rightarrow \psi \quad \text { weakly in } W^{1, r}(\Omega), r>p,
$$

also implies the same result (see [9,1]). Moreover, if

$$
\psi_{h} \leqslant \psi \quad C_{p} \text {-q.e. in } \Omega, \quad \psi_{h} \rightarrow \psi \quad C_{p} \text {-q.e. in } \Omega,
$$

then $K_{\psi_{h}}$ converges to $K_{\psi}$ in the sense of Mosco.

A necessary and sufficient condition for the convergence of $K_{\psi_{h}}$, expressed in terms of the convergence of the $C_{p}$-capacity of the level sets $\left\{x \in \Omega: \psi_{h}(x)>t\right\}$ has been given in [15].

Remark 4.5. It has been proved in [15] that if $K_{\psi_{h}}$ converges to $K_{\psi}$ in the sense of Mosco, then also $K_{T_{i}\left(\psi_{h}\right)}$ converges to $K_{T_{i}(\psi)}$ in the sense of Mosco, for every $i>0$. 
We recall now some properties of the $*$-weak and the weak convergence of measures in $\mathcal{M}_{b}(\Omega)$.

Definition 4.6. If $\mu_{h}, \mu \in \mathcal{M}_{b}(\Omega)$, we say that $\mu_{h}$ converges to $\mu *$-weakly in $\mathcal{M}_{b}(\Omega)$ if

$$
\lim _{h \rightarrow+\infty} \int_{\Omega} u \mathrm{~d} \mu_{h}=\int_{\Omega} u \mathrm{~d} \mu,
$$

for every $u \in C_{0}(\Omega)$.

For nonnegative measures we have a characterization of the $*$-weak convergence in terms of convergence of sets.

Proposition 4.7. Given $\mu_{h}, \mu \in \mathcal{M}_{b}^{+}(\Omega)$, the following conditions are equivalent:

(1) $\mu_{h}$ converges to $\mu *$-weakly in $\mathcal{M}_{b}(\Omega)$;

(2) $\mu(A) \leqslant \liminf _{h \rightarrow+\infty} \mu_{h}(A)$, for every $A$ open subset of $\Omega, \mu(K) \geqslant \limsup _{h \rightarrow+\infty} \mu_{h}(K)$, for every $K$ compact subset of $\Omega$.

Concerning the weak convergence in $\mathcal{M}_{b}(\Omega)$, the following result shows that it is stronger than the $*$-weak one.

Proposition 4.8. Given $\mu_{h}, \mu \in \mathcal{M}_{b}(\Omega)$, the following conditions are equivalent:

(1) $\mu_{h}$ converges to $\mu$ weakly in $\mathcal{M}_{b}(\Omega)$;

(2) $\lim _{h \rightarrow+\infty} \mu_{h}(B)=\mu(B)$, for every Borel set B contained in $\Omega$.

The proof of this result (see, e.g., Theorem 6.6 in [2]) relies on the Vitali-Hahn-Sacks Theorem (see, e.g., Theorem 6.4 in [2]), which is similar to the Banach-Steinhaus uniform boundedness theorem and gives a useful condition for the equiintegrability of a sequence of summable functions.

Theorem 4.9. Let $v$ be a measure in $\mathcal{M}_{b}(\Omega)$, let $g_{h}$ be a sequence in $L^{1}(\Omega, v)$ and set $\mu_{h}=g_{h} v$. Assume that, for every Borel set $B \subseteq \Omega$, the $\lim _{h \rightarrow+\infty} \mu_{h}(B)$ exists and is finite; then $g_{h}$ is equiintegrable.

In the last part of this section we give a weak notion of convergence in capacity, similar to that one considered in [10], and some properties related to it.

Definition 4.10. Let $u_{j}, u: \Omega \rightarrow \mathbb{R}$ be $C_{p}$-quasi Borel functions. We say that $u_{j}$ converges to $u$ weakly in capacity if, for every measure $\mu \in \mathcal{M}_{b, 0}^{p,+}(\Omega), u_{j}$ converges to $u$ in $\mu$-measure, i.e.,

$$
\lim _{j \rightarrow+\infty} \mu\left(\left\{x \in \Omega:\left|u_{j}(x)-u(x)\right|>\varepsilon\right\}\right)=0,
$$

for every $\varepsilon>0$.

The following proposition (see Proposition 3.5 in [10]) shows the relationship between weak convergence in $W_{0}^{1, p}(\Omega)$ and weak convergence in capacity.

Proposition 4.11. Let $u_{j}, u \in W_{0}^{1, p}(\Omega)$ be such that $u_{j}$ converges weakly to $u$ in $W_{0}^{1, p}(\Omega)$. Then $u_{j}$ converges to $u$ weakly in capacity. 
Remark 4.12. Actually, Definition 4.10 is not equivalent to Definition 3.1 of [10], where the measures $\mu$ are positive elements of $W^{-1, p^{\prime}}(\Omega)$, hence positive Radon measures (not bounded), and the convergence in $\mu$-measure is only local. However, it is easy to check that (4.4) turns out to be equivalent to the condition considered in Definition 3.1 of [10], when $\mu \in \mathcal{M}_{b}^{+}(\Omega) \cap W^{-1, p^{\prime}}(\Omega)$. On the other hand, for every measure $\mu \in \mathcal{M}_{b, 0}^{p,+}(\Omega)$ there exists a nonnegative measure $\gamma \in \mathcal{M}_{b}^{+}(\Omega) \cap W^{-1, p^{\prime}}(\Omega)$ and a nonnegative Borel measurable function $g \in$ $L^{1}(\Omega, \gamma)$ such that $\mu(A)=(g \gamma)(A)$ for every $C_{p}$-quasi open subset $A$ of $\Omega$ (see Theorem 2.2 in [14]). Hence, nothing essential changes in the proof of Proposition 3.5 in [10], when $\mu$ belongs to $\mathcal{M}_{b, 0}^{p,+}(\Omega)$.

\section{Preliminary results}

Actually, Theorem 3.6 can be improved in the following way.

Theorem 5.1. Let $\mu_{h} \in \mathcal{M}_{b, 0}^{p}(\Omega)$ and $F_{h} \in W^{-1, p^{\prime}}(\Omega)$ be such that

$$
\begin{array}{ll}
\mu_{h} \rightarrow \mu & \text { weakly in } \mathcal{M}_{b}(\Omega), \\
F_{h} \rightarrow F & \text { strongly in } W^{-1, p^{\prime}}(\Omega) ;
\end{array}
$$

let $u_{h}$ be the entropy solutions of (3.1) relative to $\mu_{h}+F_{h}$, and let $u$ be the entropy solution of (3.1) relative to $\mu+F$. Then

$$
\lim _{h \rightarrow \infty} T_{j}\left(u_{h}\right)=T_{j}(u) \quad \text { strongly in } W_{0}^{1, p}(\Omega),
$$

for every $j>0$.

Since the proof of Theorem 5.1 can be obtained by following the same scheme of the proof of Theorem 3.6, we have to enter into details only when assumption (5.1), instead of (3.13), requires some modifications. As a matter of fact, it is enough to prove here the following lemma, by which we deduce the strong convergence in $W_{0}^{1, p}(\Omega)$ of $T_{j}\left(u_{h}\right)$ to $T_{j}(u)$, exactly as in the proof of Theorem 3.6.

Lemma 5.2. Let $\mu_{h}, \mu \in \mathcal{M}_{b, 0}^{p}(\Omega)$ be such that $\mu_{h}$ converges to $\mu$ weakly in $\mathcal{M}_{b}(\Omega)$. Let $\Phi_{h}, \Phi \in W_{0}^{1, p}(\Omega) \cap$ $\mathrm{L}^{\infty}(\Omega)$ be such that $\sup _{h}\left\|\Phi_{h}\right\|_{\mathrm{L}^{\infty}(\Omega)}$ is bounded and $\Phi_{h}$ converges to $\Phi$ weakly in $W_{0}^{1, p}(\Omega)$. Then

$$
\lim _{h \rightarrow+\infty} \int_{\Omega} \Phi_{h} \mathrm{~d} \mu_{h}=\int_{\Omega} \Phi \mathrm{d} \mu .
$$

Proof. We define the measure $v \in \mathcal{M}_{b, 0}^{p,+}(\Omega)$ as

$$
v:=\sum_{h=1}^{\infty} \frac{1}{2^{h}} \frac{\left|\mu_{h}\right|}{\left|\mu_{h}\right|(\Omega)},
$$

so that $\left|\mu_{h}\right| \ll v$. This implies that $\mu_{h}=g_{h} v$, with $g_{h} \in L^{1}(\Omega, v)$; on the other hand, thanks to Proposition 4.8 , we have that $\mu_{h}(B)$ tends to $\mu(B)$, for every Borel set $B \subseteq \Omega$. Applying Theorem 4.9 we deduce that the sequence $g_{h}$ is equiintegrable, and, in conclusion, it converges to a function $g$ weakly in $L^{1}(\Omega, v)$, with $\mu=g v$. 
Now, we can prove that $\int_{\Omega} \Phi_{h} \mathrm{~d} \mu_{h}$ tends to $\int_{\Omega} \Phi \mathrm{d} \mu$, when $\Phi_{h}, \Phi$ belong to $W_{0}^{1, p}(\Omega) \cap \mathrm{L}^{\infty}(\Omega)$, with $\sup _{h}\left\|\Phi_{h}\right\|_{L^{\infty}(\Omega)}<+\infty$, and $\Phi_{h}$ converges to $\Phi$ weakly in $W_{0}^{1, p}(\Omega)$. By Proposition 4.11 , indeed, the convergence of $\Phi_{h}$ to $\Phi$ is, in particular, in $v$-measure. At this point, it is easy to obtain that

$$
\lim _{h \rightarrow+\infty} \int_{\Omega} \Phi_{h} \mathrm{~d} \mu_{h}=\lim _{h \rightarrow \infty} \int_{\Omega} \Phi_{h} g_{h} \mathrm{~d} \nu=\int_{\Omega} \Phi g \mathrm{~d} \nu=\int_{\Omega} \Phi \mathrm{d} \mu .
$$

Proposition 5.3. Assume (3.3), (3.2), (3.4), and (3.5). Let $\mu \in \mathcal{M}_{b, 0}^{p}(\Omega)$, and let $u$ be the entropy solution of (3.1). Then, for every $z \in W_{0}^{1, p}(\Omega)$ the function $u-z$ belongs to $\mathcal{T}_{0}^{1, p}(\Omega)$; more precisely, for every $j>0$, we have:

$$
\left\|T_{j}(u-z)\right\|_{W_{0}^{1, p}(\Omega)}^{p} \leqslant c(j+1),
$$

where the constant $c$ depends only on $\|\mu\|_{\mathcal{M}_{b}(\Omega)},\|z\|_{W_{0}^{1, p}(\Omega)}, p, c_{3}$, and $\|g\|_{L^{1}(\Omega)}$.

Proof. Let us consider a sequence $\mu_{n} \in W^{-1, p^{\prime}}(\Omega) \cap \mathcal{M}_{b}(\Omega)$ such that $\mu_{n}$ converges to $\mu$ strongly in $\mathcal{M}_{b}(\Omega)$. Denoting the variational solution of the problem (3.1) relative to $\mu_{n}$ by $u_{n}$, we know that $u_{n}$ tends to $u$ in the sense of Theorem 3.6. If $z \in W_{0}^{1, p}(\Omega)$, define the operator $B(v)=-\operatorname{div}(a(x, \nabla v+\nabla z)-a(x, \nabla z))$, which satisfies (3.3), (3.2), (3.4), and (3.5) with different coercitivity and growth parameters depending by $c_{2}, c_{3}, k, g, p$, and $z$. Let $v_{n}$ be the solution of

$$
\begin{cases}B(v)=\mu_{n}-A(z) & \text { in } \Omega \\ v=0 & \text { on } \partial \Omega\end{cases}
$$

that is

$$
\left\langle B\left(v_{n}\right), w\right\rangle=\left\langle\mu_{n}-A(z), w\right\rangle,
$$

or, equivalently

$$
\int_{\Omega} a\left(x, \nabla v_{n}+\nabla z\right) \nabla w \mathrm{~d} x=\left\langle\mu_{n}, w\right\rangle,
$$

for every $w \in W_{0}^{1, p}(\Omega)$. By the uniqueness of the solution of (5.4), it follows that $u_{n}=v_{n}+z$, and, since $v_{n}$ tends to the entropy solution $v$ of the problem (5.4) relative to $\mu-A z$ (see Theorem 3.6) we obtain that $u=v+z$.

At this point, the result follows by (3.8).

Remark 5.4. By the previous proposition we deduce also that if $z_{n}, z \in W_{0}^{1, p}(\Omega)$, with $z_{n}$ converging to $z$ weakly in $W_{0}^{1, p}(\Omega)$, then, for every $j>0, T_{j}\left(u-z_{n}\right)$ converges to $T_{j}(u-z)$ weakly in $W_{0}^{1, p}(\Omega)$, where $u$ is the entropy solution of (3.1) relative to $\mu \in \mathcal{M}_{b, 0}^{p}(\Omega)$.

\section{Convergence results}

The problem we deal with in this section regards the behaviour of the obstacle problems in the sense of Definition 3.8 under perturbations of the operator $A$, of the right hand side $\mu$, and of the obstacle $\psi$.

We consider a sequence $a_{h}$ of functions in $\mathcal{L}\left(c_{0}, c_{1}, \alpha, \beta\right)$, a sequence of measures $\rho_{h} \in \mathcal{M}_{b}(\Omega) \cap W^{-1, p^{\prime}}(\Omega)$, and the variational solution $u_{\rho_{h}}^{A_{h}}$ of

$$
\left\{\begin{array}{l}
A_{h}\left(u_{\rho_{h}}^{A_{h}}\right)=\rho_{h} \quad \text { in } \Omega \\
u_{\rho_{h}}^{A_{h}} \in W_{0}^{1, p}(\Omega) .
\end{array}\right.
$$


We assume that

$$
\sup _{h}\left\|\rho_{h}\right\|_{\mathcal{M}_{b}(\Omega)}<+\infty
$$

and that the function $\psi_{h}$ satisfies:

$$
\psi_{h} \leqslant u_{\rho_{h}}^{A_{h}} \quad C_{p} \text {-q.e. in } \Omega .
$$

Moreover we suppose that

$$
\psi \leqslant 0 \quad C_{p} \text {-q.e. in } \Omega .
$$

We can now state the main result of this section.

Theorem 6.1. Let $a_{h}$ be a sequence in $\mathcal{L}\left(c_{0}, c_{1}, \alpha, \beta\right)$, which $G$-converges to a function $a$, and let $A_{h}$ and $A$ be the operators associated to $a_{h}$ and $a$, respectively. Let us assume (6.1), (6.2), and (6.3), with $K_{\psi_{h}}$ converging to $K_{\psi}$ in the sense of Mosco. Finally, consider $\mu_{h}, \mu \in \mathcal{M}_{b, 0}^{p}(\Omega)$, with $\mu_{h}$ converging to $\mu$ weakly in $\mathcal{M}_{b}(\Omega)$. Then the solutions $u_{h}$ and $u$ of the obstacle problems $O P\left(A_{h}, \mu_{h}, \psi_{h}\right)$ and $O P(A, \mu, \psi)$, respectively, satisfy

$$
\begin{aligned}
& T_{j}\left(u_{h}\right) \rightarrow T_{j}(u) \quad \text { weakly in } W_{0}^{1, p}(\Omega), \quad \text { for every } j>0, \\
& a_{h}\left(x, \nabla u_{h}\right) \rightarrow a(x, \nabla u) \quad \text { weakly in } L^{q}(\Omega)^{N}, \quad \text { for every } q<\frac{N}{N-1}, \\
& \int_{\Omega} a_{h}\left(x, \nabla u_{h}\right) \nabla T_{j}\left(u_{h}\right) \mathrm{d} x \rightarrow \int_{\Omega} a(x, \nabla u) \nabla T_{j}(u) \mathrm{d} x, \quad \text { for every } j>0 .
\end{aligned}
$$

Remark 6.2. By formal modifications we can prove Theorem 6.1 replacing (6.3) with (3.18) and

$$
\psi \leqslant M \quad C_{p} \text {-q.e. in } \Omega,
$$

where $M$ is a positive constant.

Proof of Theorem 6.1. To simplify the exposition, it is convenient to divide the proof into various steps.

Step 1. We will prove (6.4).

Proof of Step 1. Let us recall that the solution $u_{h}$ of the obstacle problem $\operatorname{OP}\left(A_{h}, \mu_{h}, \psi_{h}\right)$ is the entropy solution of Eq. (4.1) relative to $\mu+\lambda_{h}$, i.e., for every $\varphi \in W_{0}^{1, p}(\Omega) \cap L^{\infty}(\Omega), u_{h}$ satisfies:

$$
\int_{\Omega} a_{h}\left(x, \nabla u_{h}\right) \nabla T_{j}\left(u_{h}-\varphi\right) \mathrm{d} x=\int_{\Omega} T_{j}\left(u_{h}-\varphi\right) \mathrm{d} \mu_{h}+\int_{\Omega} T_{j}\left(u_{h}-\varphi\right) \mathrm{d} \lambda_{h},
$$

where the obstacle reaction $\lambda_{h} \in \mathcal{M}_{b, 0}^{p,+}(\Omega)$ satisfies (3.19), i.e.

$$
\left\|\lambda_{h}\right\|_{\mathcal{M}_{b}(\Omega)} \leqslant\left\|\left(\mu_{h}-\rho_{h}\right)^{-}\right\|_{\mathcal{M}_{b}(\Omega)} .
$$

Combining the previous estimate with (6.1) and (3.8), we obtain that, for every $j>0$,

$$
\int_{\Omega}\left|\nabla T_{j}\left(u_{h}\right)\right|^{p} \mathrm{~d} x \leqslant c j
$$

where the constant $c$ does not depend on $j$ and $h$. Working as in the proof of Theorem 6.1 of [3] we have that there exists a subsequence of $u_{h}$ (still denoted by $u_{h}$ ) and a function $u^{*} \in \mathcal{T}_{0}^{1, p}(\Omega)$ such that $u_{h}$ converges to $u^{*}$ a.e. in $\Omega$ and, for every $j>0, T_{j}\left(u_{h}\right)$ converges to $T_{j}\left(u^{*}\right)$ weakly in $W_{0}^{1, p}(\Omega)$. Since also $K_{T_{j}\left(\psi_{h}\right)}$ converges to $K_{T_{j}(\psi)}$ in the sense of Mosco (see Remark 4.5), by the weakly convergence in $W_{0}^{1, p}(\Omega)$ of $T_{j}\left(u_{h}\right)$ to $T_{j}\left(u^{*}\right)$ we 
deduce that $T_{j}\left(u^{*}\right) \geqslant T_{j}(\psi) C_{p}$-q.e. in $\Omega$, for every $j>0$, so that $u^{*} \geqslant \psi C_{p}$-q.e. in $\Omega$. Furthermore, by the weak convergence in $W_{0}^{1, p}(\Omega)$ of $T_{j}\left(u_{h}\right)$ to $T_{j}(u)$, we obtain that also $T_{j}\left(u^{*}\right)$ satisfies (6.8), which implies (3.9) for $u^{*}$.

Let us consider a function $\Phi \in W_{0}^{1, p}(\Omega)$, with $\Phi \geqslant \psi$, and the solution $w_{h}$ of the variational inequality $\operatorname{VI}\left(A_{h}, A(\Phi), \psi_{h}\right)$. By Theorem 3.1 of [16], $w_{h}$ satisfies:

$$
\begin{aligned}
& w_{h} \rightarrow w \quad \text { weakly in } W_{0}^{1, p}(\Omega), \\
& a_{h}\left(x, \nabla w_{h}\right) \rightarrow a(x, \nabla w) \quad \text { weakly in } L^{p^{\prime}}(\Omega)^{N}, \\
& \left\langle a_{h}\left(x, \nabla w_{h}\right), w_{h}\right\rangle \rightarrow\langle a(x, \nabla w), w\rangle,
\end{aligned}
$$

where $w$ is the solution of $\operatorname{VI}(A, A(\Phi), \psi)$; so that $w=\Phi$ (see Characterization 1).

Moreover, $w_{h}$ satisfies

$$
\int_{\Omega} a_{h}\left(x, \nabla w_{h}\right) \nabla\left(w_{h}-v\right) \mathrm{d} x \leqslant\left\langle A(\Phi), w_{h}-v\right\rangle=\int_{\Omega} a(x, \nabla \Phi) \nabla\left(w_{h}-v\right),
$$

for every $v \in W_{0}^{1, p}(\Omega)$, with $v \geqslant \psi_{h}$. Now, using the monotonicity of the operator $A_{h}$, we can rewrite (6.7) as

$$
\int_{\Omega} a_{h}(x, \nabla \varphi) \nabla T_{j}\left(u_{h}-\varphi\right) \mathrm{d} x \leqslant \int_{\Omega} T_{j}\left(u_{h}-\varphi\right) \mathrm{d} \mu_{h}+\int_{\Omega} T_{j}\left(u_{h}-\varphi\right) \mathrm{d} \lambda_{h},
$$

for every $\varphi \in W_{0}^{1, p}(\Omega) \cap \mathrm{L}^{\infty}(\Omega)$. We would like to use $w_{h}$ in (6.10), but, a priori, we do not know that $w_{h}$ is a bounded function. Let us note, nevertheless, that if a function $\varphi$ is in $W_{0}^{1, p}(\Omega)$, for every $i>0$, we can use $T_{i}(\varphi)$ as function test in (6.10). Observe now that, letting $i$ tend to infinity, $T_{i}(\varphi)$ converges to $\varphi$ strongly in $W_{0}^{1, p}(\Omega)$, so that, on one hand $a_{h}\left(x, \nabla T_{i}(\varphi)\right)$ tends to $a_{h}(x, \nabla \varphi)$ strongly in $L^{p^{\prime}}(\Omega)^{N}$, on the other $T_{j}\left(u_{h}-T_{i}(\varphi)\right)$ converges to $T_{j}\left(u_{h}-\varphi\right)$ weakly in $W_{0}^{1, p}(\Omega)$, as observed in Remark 5.4. Now we can rewrite (6.10) for every $\varphi \in W_{0}^{1, p}(\Omega)$, and, in particular, choosing $w_{h}$ as function test we obtain

$$
\begin{aligned}
\int_{\Omega} a_{h}\left(x, \nabla w_{h}\right) \nabla T_{j}\left(u_{h}-w_{h}\right) \mathrm{d} x & \leqslant \int_{\Omega} T_{j}\left(u_{h}-w_{h}\right) \mathrm{d} \mu_{h}+\int_{\Omega} T_{j}\left(u_{h}-w_{h}\right) \mathrm{d} \lambda_{h} \\
& \leqslant \int_{\Omega} T_{j}\left(u_{h}-w_{h}\right) \mathrm{d} \mu_{h},
\end{aligned}
$$

where the last inequality follows by the complementarity system (3.21) and by the fact that $w_{h} \geqslant \psi_{h} C_{p}$-q.e. in $\Omega$.

The choice of the function $v=v_{h}:=w_{h}-T_{j}\left(w_{h}-u_{h}\right)$ as test in (6.9) is admissible and gives:

$$
\int_{\Omega} a_{h}\left(x, \nabla w_{h}\right) \nabla T_{j}\left(w_{h}-u_{h}\right) \mathrm{d} x \leqslant \int_{\Omega} a(x, \nabla \Phi) \nabla T_{j}\left(w_{h}-u_{h}\right) \mathrm{d} x,
$$

which, with (6.11), implies

$$
\int_{\Omega} a(x, \nabla \Phi) \nabla T_{j}\left(u_{h}-w_{h}\right) \mathrm{d} x \leqslant \int_{\Omega} T_{j}\left(u_{h}-w_{h}\right) \mathrm{d} \mu_{h} .
$$

By the estimate (5.3), it is easy to prove that $T_{j}\left(u_{h}-w_{h}\right)$ converges to $T_{j}(u-\Phi)$ weakly in $W_{0}^{1, p}(\Omega)$, and, thanks to Lemma 5.2 we easily pass to the limit in (6.13). In conclusion, we obtain

$$
\int_{\Omega} a(x, \nabla \Phi) \nabla T_{j}\left(u^{*}-\Phi\right) \mathrm{d} x \leqslant \int_{\Omega} T_{j}\left(u^{*}-\Phi\right) \mathrm{d} \mu,
$$

for every $\Phi \in W_{0}^{1, p}(\Omega), \Phi \geqslant \psi C_{p}$-q.e. in $\Omega$. 
Thanks to the next lemma, we have the following fact:

$$
\int_{\Omega} a\left(x, \nabla u^{*}\right) \nabla T_{j}\left(u^{*}-\varphi\right) \mathrm{d} x \leqslant \int_{\Omega} T_{j}\left(u^{*}-\varphi\right) \mathrm{d} \mu,
$$

for every $\varphi \in W_{0}^{1, p}(\Omega) \cap \mathrm{L}^{\infty}(\Omega), \varphi \geqslant \psi C_{p}$-q.e. in $\Omega$. As observed in Remark 3.11, the previous formulation characterizes uniquely the function $u^{*}$. Thus, having denoted the solution of $O P(A, \mu, \psi)$ by $u$, we have $u^{*}=u$; this implies that the whole sequence $T_{j}\left(u_{h}\right)$ (and not only a subsequence) converge to $T_{j}(u)$.

Hence, to conclude, we have to prove the following lemma, which is inspired by Lemma 1.2 of [4]. We give here the proof for the sake of completeness.

Lemma 6.3. Assume $\mu$ be in $\mathcal{M}_{b, 0}^{p}(\Omega)$ and $\psi$ satisfy (6.3). Under hypotheses (3.2), (3.3), (3.4), and (3.5) a solution $u$ of

$$
\left\{\begin{array}{l}
u \in \mathcal{T}_{0}^{1, p}(\Omega), \quad u \geqslant \psi, \\
\int_{\Omega} a(x, \nabla \Phi) \nabla T_{j}(u-\Phi) \mathrm{d} x \leqslant \int_{\Omega} T_{j}(u-\Phi) \mathrm{d} \mu, \\
\forall j>0, \forall \Phi \in W_{0}^{1, p}(\Omega), \quad \Phi \geqslant \psi,
\end{array}\right.
$$

satisfying (3.8), is also a solution of

$$
\left\{\begin{array}{l}
u \in \mathcal{T}_{0}^{1, p}(\Omega), \quad u \geqslant \psi, \\
\int_{\Omega} a(x, \nabla u) \nabla T_{k}(u-\varphi) \mathrm{d} x \leqslant \int_{\Omega} T_{k}(u-\varphi) \mathrm{d} \mu, \\
\forall k>0, \forall \varphi \in W_{0}^{1, p}(\Omega) \cap \mathrm{L}^{\infty}(\Omega), \quad \varphi \geqslant \psi .
\end{array}\right.
$$

The converse is also true.

Proof. Let $u$ be a solution of (6.15) and $\varphi \in W_{0}^{1, p}(\Omega) \cap \mathrm{L}^{\infty}(\Omega), \varphi \geqslant \psi$. The choice of $\Phi=t T_{i}(u)+(1-t) \varphi$, with $i>0$ and $t \in(0,1)$, in $(6.15)$ is admissible and gives

$$
\left\{\begin{array}{l}
I_{i} \leqslant J_{i} \\
I_{i}=\int_{\Omega} a\left(x, t \nabla T_{i}(u)+(1-t) \nabla \varphi\right) \nabla T_{j}\left(u-t T_{i}(u)-(1-t) \varphi\right) \mathrm{d} x, \\
J_{i}=\int_{\Omega} T_{j}\left(u-t T_{i}(u)-(1-t) \varphi\right) \mathrm{d} \mu
\end{array}\right.
$$

Now,

$$
\begin{aligned}
I_{i}= & \int_{\{|u| \leqslant i\}} a(x, t \nabla u+(1-t) \nabla \varphi) \nabla T_{j}((1-t)(u-\varphi)) \mathrm{d} x \\
& +\int_{\{|u|>i\} \cap\left\{\left|u-t T_{i}(u)-(1-t) \varphi\right| \leqslant j\right\}} a(x,(1-t) \nabla \varphi) \nabla(u-(1-t) \varphi) \mathrm{d} x,
\end{aligned}
$$

since $\nabla T_{j}\left(u-t T_{i}(u)-(1-t) \varphi\right)=0$ where $\left|u-t T_{i}(u)-(1-t) \varphi\right|>j$. The set $\{|u|>i\} \cap\left\{\mid u-t T_{i}(u)-(1-\right.$ $t) \varphi \mid \leqslant j\}$ is empty if we choose $i>\|\varphi\|_{\mathrm{L}^{\infty}(\Omega)}$ and $0<j \leqslant(1-t)\left(i-\|\varphi\|_{\mathrm{L}^{\infty}(\Omega)}\right)$; hence

$$
I_{i}=\int_{\{|u| \leqslant i\}} a(x, t \nabla u+(1-t) \nabla \varphi) \nabla T_{j}((1-t)(u-\varphi)) \mathrm{d} x .
$$

Let us consider $J_{i}$ : 


$$
\begin{aligned}
J_{i} & =\int_{\{|u| \leqslant i\}} T_{j}((1-t)(u-\varphi)) \mathrm{d} \mu+\int_{\{|u|>i\}} T_{j}\left(u-t T_{i}(u)-(1-t) \varphi\right) \mathrm{d} \mu \\
& \leqslant \int_{\{|u| \leqslant i\}} T_{j}((1-t)(u-\varphi)) \mathrm{d} \mu+j|\mu|(\{|u|>i\}) .
\end{aligned}
$$

Now we pass to the limit as $i$ tends to $+\infty$ in (6.17); taking into account (3.9), we obtain, by the previous remarks about $I_{i}$ and $J_{i}$ that

$$
\begin{aligned}
I & :=\lim _{i \rightarrow+\infty} I_{i}=\int_{\Omega} a(x, t \nabla u+(1-t) \nabla \varphi) \nabla T_{j}((1-t)(u-\varphi)) \mathrm{d} x \\
& \leqslant \lim _{i \rightarrow \infty} J_{i}=\int_{\Omega} T_{j}((1-t)(u-\varphi)) \mathrm{d} \mu=: J,
\end{aligned}
$$

for every $j>0$. Let us write $I$ as

$$
I=(1-t) \int_{\{(1-t)|u-\varphi| \leqslant j\}} a(x, t \nabla u+(1-t) \nabla \varphi) \nabla(u-\varphi) \mathrm{d} x,
$$

while

$$
J=(1-t) \int_{\{(1-t)|u-\varphi| \leqslant j\}}(u-\varphi) \mathrm{d} \mu+\int_{\{(1-t)(u-\varphi)>j\}} j \mathrm{~d} \mu+\int_{\{(1-t)(u-\varphi)<-j\}}(-j) \mathrm{d} \mu .
$$

Let $k>0$ and $j$ such that $j=k(1-t)$, so that $I \leqslant J$ implies

$$
(1-t) \int_{\Omega} a(x, t \nabla u+(1-t) \nabla \varphi) \nabla T_{k}(u-\varphi) \mathrm{d} x \leqslant(1-t) \int_{\Omega} T_{k}(u-\varphi) \mathrm{d} \mu .
$$

Dividing by $(1-t)$ and passing to the limit with respect to $t \rightarrow 1^{-}$, we obtain (6.16).

The converse is just the monotonicity of the operator $A$. Let us note that, if $u$ solves (6.16), then $u$ satisfies (3.8), since we can use $\varphi=0$ as test in (6.16) and use (3.3).

Step 2. Denoting the obstacle reactions of $u_{h}$ and $u$ by $\lambda_{h}$ and $\lambda$, respectively, we will prove that

$$
\lim _{h \rightarrow+\infty} \int_{\Omega} \Phi_{h} \mathrm{~d} \lambda_{h}=\int_{\Omega} \Phi \mathrm{d} \lambda
$$

for every $\Phi \in W_{0}^{1, q^{\prime}}(\Omega)$, with $q<\frac{N}{N-1}$, and for every $\Phi_{h} \in W_{0}^{1, p}(\Omega) \cap \mathrm{L}^{\infty}(\Omega)$, with $\sup _{h}\left\|\Phi_{h}\right\|_{\mathrm{L}^{\infty}(\Omega)}<+\infty$, converging to $\Phi$ strongly in $W_{0}^{1, p}(\Omega)$.

Proof of Step 2. For every $i>0$ and for every $t \in \mathbb{R}, t \neq 0$, we consider the solution $v_{h}$ of the variational inequality $\operatorname{VI}\left(A_{h}, A\left(T_{i}(u)+t \Phi_{h}\right), \psi_{h}+t \Phi_{h}\right)$ and the obstacle reaction $\eta_{h}$ associated with it. Observing that $A\left(T_{i}(u)+t \Phi_{h}\right)$ converges to $A\left(T_{i}(u)+t \Phi\right)$ strongly in $W^{-1, p^{\prime}}(\Omega)$, and that $K_{\psi_{h}+t \Phi_{h}}$ converges to $K_{\psi+t \Phi}$ in the sense of Mosco, we can apply Theorem 3.1 of [16] to deduce:

$$
\begin{array}{ll}
v_{h} \rightarrow v & \text { weakly in } W_{0}^{1, p}(\Omega), \\
\eta_{h} \rightarrow \eta & \text { weakly in } W^{-1, p^{\prime}}(\Omega),
\end{array}
$$

where $v$ is the solution of $\operatorname{VI}\left(A, A\left(T_{i}(u)+t \Phi\right), \psi+t \Phi\right)$ and $\eta$ is the obstacle reaction associated with it. On the other hand, thanks to (6.3), for every $i>0$, we have that $T_{i}(u) \geqslant \psi C_{p}$-q.e. in $\Omega$, so that $v=T_{i}(u)+t \Phi$ and $\eta=0$ (see Characterization 1). 
Consider now, for every $l>0$ and for every $j>0$, the inequality

$$
\int_{\Omega}\left(a_{h}\left(x, \nabla u_{h}\right)-a_{h}\left(x, \nabla T_{l}\left(v_{h}\right)\right)\right) \nabla T_{j}\left(u_{h}-T_{l}\left(v_{h}\right)\right) \mathrm{d} x \geqslant 0,
$$

which follows by the monotonicity of $a_{h}$. If we use the entropy formulation of $u_{h}$ in the previous inequality we obtain

$$
\int_{\Omega} T_{j}\left(u_{h}-T_{l}\left(v_{h}\right)\right) \mathrm{d} \lambda_{h}+\int_{\Omega} T_{j}\left(u_{h}-T_{l}\left(v_{h}\right)\right) \mathrm{d} \mu_{h} \geqslant \int_{\Omega} a_{h}\left(x, \nabla T_{l}\left(v_{h}\right)\right) \nabla T_{j}\left(u_{h}-T_{l}\left(v_{h}\right)\right) \mathrm{d} x ;
$$

passing to the limit as $l$ tends to $+\infty$ thanks to Proposition 5.3 (see also Remark 5.4), and using the variational formulation (3.15) satisfied by $v_{h}$, we rewrite (6.19) as

$$
\left\{\begin{array}{l}
I_{h}+I I_{h} \geqslant I I I_{h}+I V_{h}, \\
I_{h}=\int_{\Omega} T_{j}\left(u_{h}-v_{h}\right) \mathrm{d} \lambda_{h}, \\
I I_{h}=\int_{\Omega} T_{j}\left(u_{h}-v_{h}\right) \mathrm{d} \mu_{h}, \\
I I I_{h}=\int_{\Omega} a\left(x, \nabla\left(T_{i}(u)+t \Phi_{h}\right)\right) \nabla T_{j}\left(u_{h}-v_{h}\right) \mathrm{d} x, \\
I V_{h}=\left\langle\eta_{h}, T_{j}\left(u_{h}-v_{h}\right)\right\rangle .
\end{array}\right.
$$

By the complementarity system (3.16), we have that

$$
I V_{h}=\int_{\Omega} T_{j}\left(u_{h}-\psi_{h}-t \Phi_{h}\right) \mathrm{d} \eta_{h} \geqslant \int_{\Omega} T_{j}\left(-t \Phi_{h}\right) \mathrm{d} \eta_{h}=\left\langle\eta_{h}, T_{j}\left(-\Phi_{h}\right)\right\rangle,
$$

which tends to 0 as $h$ goes to $+\infty$, i.e.

$$
\liminf _{h \rightarrow+\infty} I V_{h} \geqslant 0 .
$$

Moreover, by (5.3), it is easy to check that $T_{j}\left(u_{h}-v_{h}\right)$ converges to $T_{j}\left(u-T_{i}(u)-t \Phi\right)$ weakly in $W_{0}^{1, p}(\Omega)$, so that we can apply Lemma 5.2 to deduce that

$$
\lim _{h \rightarrow+\infty} I I_{h}=\int_{\Omega} T_{j}\left(u-T_{i}(u)-t \Phi\right) \mathrm{d} \mu .
$$

Since, thanks to (2.1), $a\left(x, \nabla\left(T_{i}(u)+t \Phi_{h}\right)\right)$ converges to $a\left(x, \nabla\left(T_{i}(u)+t \Phi\right)\right)$ strongly in $L^{p^{\prime}}(\Omega)^{N}$, we pass to the limit also in $I I I_{h}$, obtaining

$$
\lim _{h \rightarrow+\infty} I I I_{h}=\int_{\Omega} a\left(x, \nabla\left(T_{i}(u)+t \Phi\right)\right) \nabla T_{j}\left(u-T_{i}(u)-t \Phi\right) \mathrm{d} x .
$$

Combining (6.21), (6.22) and (6.23) we have

$$
\begin{gathered}
\liminf _{h \rightarrow+\infty} \int_{\Omega} T_{j}\left(u_{h}-v_{h}\right) \mathrm{d} \lambda_{h}+\int_{\Omega} T_{j}\left(u-T_{i}(u)-t \Phi\right) \mathrm{d} \mu \\
\geqslant \int_{\Omega} a\left(x, \nabla\left(T_{i}(u)+t \Phi\right)\right) \nabla T_{j}\left(u-T_{i}(u)-t \Phi\right) \mathrm{d} x,
\end{gathered}
$$

which can be written also as

$$
\left\{\begin{array}{l}
\liminf _{h \rightarrow+\infty} I_{h}-I^{i} \geqslant I I^{i} \\
I^{i}=\int_{\Omega} T_{j}\left(u-T_{i}(u)-t \Phi\right) \mathrm{d} \lambda \\
I I^{i}=\int_{\Omega}\left(a\left(x, \nabla\left(T_{i}(u)+t \Phi\right)\right)-a(x, \nabla u)\right) \nabla T_{j}\left(u-T_{i}(u)-t \Phi\right) \mathrm{d} x,
\end{array}\right.
$$


using the entropy formulation satisfied by $u$. By the complementarity system (3.21), we have that

$$
\liminf _{h \rightarrow+\infty} I_{h}=\liminf _{h \rightarrow+\infty} \int_{\Omega} T_{j}\left(\psi_{h}-v_{h}\right) \mathrm{d} \lambda_{h} ;
$$

on the other hand, since $v_{h} \geqslant \psi_{h}+t \Phi_{h}$ we obtain by the previous equality that

$$
\liminf _{h \rightarrow+\infty} I_{h} \leqslant \liminf _{h \rightarrow+\infty} \int_{\Omega} T_{j}\left(-t \Phi_{h}\right) \mathrm{d} \lambda_{h} .
$$

On the other hand, thanks to (3.9) it is easy to check that

$$
\lim _{i \rightarrow+\infty} I^{i}=\int_{\Omega} T_{j}(-t \Phi) \mathrm{d} \lambda .
$$

Finally, in $I I^{i}$ we split the integral into the sets where $|u| \leqslant i$ and where $|u|>i$, getting

$$
\begin{aligned}
I I^{i}= & \int_{\{|u| \leqslant i\}}(a(x, \nabla(u+t \Phi))-a(x, \nabla u)) \nabla T_{j}(-t \Phi) \mathrm{d} x \\
& +\int_{\{|u|>i\} \cap\left\{\left|u-T_{i}(u)-t \Phi\right| \leqslant j\right\}}(a(x, \nabla(t \Phi))-a(x, \nabla u)) \nabla(u-t \Phi) \mathrm{d} x,
\end{aligned}
$$

since $\nabla T_{j}\left(u-T_{i}(u)-t \Phi\right)=0$ where $\left|u-T_{i}(u)-t \Phi\right|>j$. Let us observe that $\left\{\left|u-T_{i}(u)-t \Phi\right| \leqslant j\right\} \subseteq\{|u| \leqslant$ $\left.i+j+|t|\|\Phi\|_{\mathrm{L}^{\infty}(\Omega)}\right\}$, so that, by the growth conditions assumed on $a$ and by (3.10), it is easy to prove that

$$
\lim _{i \rightarrow+\infty} \int_{\{|u|>i\} \cap\left\{\left|u-T_{i}(u)-t \Phi\right| \leqslant j\right\}}(a(x, \nabla(t \Phi))-a(x, \nabla u)) \nabla(u-t \Phi) \mathrm{d} x=0,
$$

as well as

$$
\begin{aligned}
& \lim _{i \rightarrow+\infty} \int_{\{|u| \leqslant i\}}(a(x, \nabla(u+t \Phi))-a(x, \nabla u)) \nabla T_{j}(-t \Phi) \mathrm{d} x \\
& =\int_{\Omega}(a(x, \nabla(u+t \Phi))-a(x, \nabla u)) \nabla T_{j}(-t \Phi) \mathrm{d} x,
\end{aligned}
$$

since $a(x, \nabla(u+t \Phi))-a(x, \nabla u) \in L^{q}(\Omega)^{N}$ and, by hypothesis, $\Phi \in W_{0}^{1, q^{\prime}}(\Omega)$. Combining (6.26)-(6.28) and (6.29) we have

$$
\liminf _{h \rightarrow+\infty} \int_{\Omega} T_{j}\left(-t \Phi_{h}\right) \mathrm{d} \lambda_{h}-\int_{\Omega} T_{j}(-\Phi) \mathrm{d} \lambda \geqslant \int_{\Omega}(a(x, \nabla(u+t \Phi))-a(x, \nabla u)) \nabla T_{j}(-t \Phi) \mathrm{d} x,
$$

and, for $j \geqslant|t|\left(\left\|\Phi_{h}\right\|_{\mathrm{L}^{\infty}(\Omega)} \vee\|\Phi\|_{\mathrm{L}^{\infty}(\Omega)}\right)$

$$
\liminf _{h \rightarrow+\infty} \int_{\Omega}-t \Phi_{h} \mathrm{~d} \lambda_{h}+t \int_{\Omega} \Phi \mathrm{d} \lambda \geqslant-t \int_{\Omega}(a(x, \nabla(u+t \Phi))-a(x, \nabla u)) \nabla \Phi \mathrm{d} x .
$$

At this point, dividing by $|t|$ and passing to the limit with respect to $t \rightarrow 0$, we obtain (6.18).

Step 3. We will prove (6.5). 
Proof of Step 3. We recall that $u_{h}$ satisfies (3.11), i.e.

$$
\int_{\Omega} a_{h}\left(x, \nabla u_{h}\right) \nabla \Phi \mathrm{d} x=\int_{\Omega} \Phi \mathrm{d} \mu_{h}+\int_{\Omega} \Phi \mathrm{d} \lambda_{h},
$$

for every $\Phi \in W_{0}^{1, q^{\prime}}(\Omega)$, with $1<q<\frac{N}{N-1}$. We just observed that $W_{0}^{1, q^{\prime}}(\Omega) \subseteq C(\bar{\Omega})$, so that, thanks to Lemma 5.2 and (6.18), we can pass to the limit as $h$ goes to $+\infty$ in the last two terms of (6.30), obtaining

$$
\lim _{h \rightarrow+\infty} \int_{\Omega} a_{h}\left(x, \nabla u_{h}\right) \nabla \Phi \mathrm{d} x=\int_{\Omega} \Phi \mathrm{d} \mu+\int_{\Omega} \Phi \mathrm{d} \lambda=\int_{\Omega} a(x, \nabla u) \nabla \Phi \mathrm{d} x,
$$

where the last equality follows by Eq. (3.11) satisfied by $u$. In other words, we proved that

$$
-\operatorname{div}\left(a_{h}\left(x, \nabla u_{h}\right)\right) \rightarrow-\operatorname{div}(a(x, \nabla u)) \quad \text { weakly in } W^{-1, q}(\Omega), \text { for every } q<\frac{N}{N-1} .
$$

On the other hand, $a_{h}\left(x, \nabla u_{h}\right)$ is equibounded (with respect to $h$ ) in the $L^{q}$-norm, as observed in Remark 3.5. By this fact we easily deduce that

$$
a_{h}\left(x, \nabla u_{h}\right) \rightarrow \sigma \text { weakly in } L^{q}(\Omega)^{N},
$$

where $\operatorname{div}(a(x, \nabla u)-\sigma)=0$. As we will see later, to prove (6.5), it is enough to show, by Minty's trick, that

$$
\lim _{h \rightarrow+\infty} \int_{\Omega} a_{h}\left(x, \nabla u_{h}\right) \nabla \Phi \phi \mathrm{d} x=\int_{\Omega} a(x, \nabla u) \nabla \Phi \phi \mathrm{d} x,
$$

for every $\Phi \in W_{0}^{1, q^{\prime}}(\Omega)$ and for every $\phi \in C^{1}(\bar{\Omega})$.

With minor changes with respect to the proof of Step 2, we will prove (6.32). Let $\Phi \in W_{0}^{1, q^{\prime}}(\Omega)$ and $t \in \mathbb{R}$, with $t \neq 0$; then the solution $v_{h}$ of $\operatorname{VI}\left(A_{h}, A\left(T_{i}(u)+t \Phi\right), \psi_{h}+t \Phi\right)$ and the obstacle reaction $\eta_{h}$ associated with it are such that

$$
\begin{aligned}
& v_{h} \rightarrow T_{i}(u)+t \Phi \quad \text { weakly in } W_{0}^{1, p}(\Omega), \\
& a_{h}\left(x, \nabla v_{h}\right) \rightarrow a\left(x, \nabla\left(T_{i}(u)+t \Phi\right)\right) \quad \text { weakly in } L^{p^{\prime}}(\Omega)^{N}, \\
& \eta_{h} \rightarrow 0 \quad \text { weakly in } W^{-1, p^{\prime}}(\Omega),
\end{aligned}
$$

since $T_{i}(u)+t \Phi$ is the solution of $\operatorname{VI}\left(A, A\left(T_{i}(u)+t \Phi\right), \psi+t \Phi\right)$. By the monotonicity assumption on $a_{h}(x, \cdot)$ we have, for every $l, j>0$

$$
\int_{\Omega}\left(a_{h}\left(x, \nabla u_{h}\right)-a_{h}\left(x, \nabla T_{l}\left(v_{h}\right)\right)\right) \nabla T_{j}\left(u_{h}-T_{l}\left(v_{h}\right)\right) \phi \mathrm{d} x \geqslant 0,
$$

where $\phi \in C^{1}(\bar{\Omega})$, with $\phi \geqslant 0$. For convenience we write the previous inequality in the form

$$
\begin{gathered}
\int_{\Omega}\left(a_{h}\left(x, \nabla u_{h}\right)-a_{h}\left(x, \nabla T_{l}\left(v_{h}\right)\right)\right) \nabla\left(T_{j}\left(u_{h}-T_{l}\left(v_{h}\right)\right) \phi\right) \mathrm{d} x \\
\geqslant \int_{\Omega}\left(a_{h}\left(x, \nabla u_{h}\right)-a_{h}\left(x, \nabla T_{l}\left(v_{h}\right)\right)\right) \nabla \phi T_{j}\left(u_{h}-T_{l}\left(v_{h}\right)\right) \mathrm{d} x,
\end{gathered}
$$


which gives, using the entropy formulation (3.12) of $u_{h}$ and letting $l$ tend to $+\infty$, as in the proof of Step 2,

$$
\left\{\begin{array}{l}
I_{h}+I I_{h}+I I I_{h} \geqslant I V_{h}+V_{h}, \\
I_{h}=\int_{\Omega} T_{j}\left(u_{h}-v_{h}\right) \phi \mathrm{d} \lambda_{h}, \\
I I_{h}=\int_{\Omega} T_{j}\left(u_{h}-v_{h}\right) \phi \mathrm{d} \mu_{h}, \\
I I I_{h}=-\int_{\Omega} a_{h}\left(x, \nabla u_{h}\right) \nabla \phi T_{j}\left(u_{h}-v_{h}\right) \mathrm{d} x, \\
I V_{h}=\int_{\Omega} a_{h}\left(x, \nabla v_{h}\right) \nabla\left(T_{j}\left(u_{h}-v_{h}\right) \phi\right) \mathrm{d} x, \\
V_{h}=-\int_{\Omega} a_{h}\left(x, \nabla v_{h}\right) \nabla \phi T_{j}\left(u_{h}-v_{h}\right) \mathrm{d} x .
\end{array}\right.
$$

The same tools used to deduce (6.26) give:

$$
I_{h} \leqslant \int_{\Omega} T_{j}(-t \Phi) \phi \mathrm{d} \lambda_{h}
$$

choosing $j \geqslant|t|\|\Phi\|_{\mathrm{L}^{\infty}(\Omega)}$ and using the formulation (3.11) satisfied by $u_{h}$, we have:

$$
I_{h} \leqslant-t \int_{\Omega} a_{h}\left(x, \nabla u_{h}\right) \nabla(\Phi \phi) \mathrm{d} x+t \int_{\Omega} \Phi \phi \mathrm{d} \mu_{h} .
$$

Thanks to the variational formulation satisfied by $v_{h}$ we write $I V_{h}$ as

$$
I V_{h}=\int_{\Omega} a\left(x, \nabla\left(T_{i}(u)+t \Phi\right)\right) \nabla\left(T_{j}\left(u_{h}-v_{h}\right) \phi\right) \mathrm{d} x+\left\langle\eta_{h}, T_{j}\left(u_{h}-v_{h}\right) \phi\right\rangle
$$

and we obtain that

$$
\liminf _{h \rightarrow+\infty} I V_{h} \geqslant \int_{\Omega} a\left(x, \nabla\left(T_{i}(u)+t \Phi\right)\right) \nabla\left(T_{j}\left(u-T_{i}(u)-t \Phi\right) \phi\right) \mathrm{d} x,
$$

since we can work as in the proof of (6.21) and (6.23). Analogously, as we prove (6.22), we have also that

$$
\lim _{h \rightarrow+\infty} I I_{h}=\int_{\Omega} T_{j}\left(u-T_{i}(u)-t \Phi\right) \phi \mathrm{d} \mu .
$$

On the other hand, it is easy to check that

$$
\lim _{h \rightarrow+\infty} I I I_{h}=t \lim _{h \rightarrow+\infty} \int_{\Omega} a_{h}\left(x, \nabla u_{h}\right) \nabla \phi \Phi \mathrm{d} x+\int_{\Omega} \sigma \nabla \phi\left(-t \Phi-T_{j}\left(u-T_{i}(u)-t \Phi\right)\right) \mathrm{d} x,
$$

as well as

$$
\lim _{h \rightarrow+\infty} V_{h}=-\int_{\Omega} a\left(x, \nabla\left(T_{i}(u)+t \Phi\right)\right) \nabla \phi T_{j}\left(u-T_{i}(u)-t \Phi\right) \mathrm{d} x .
$$

Combining (6.33)-(6.36) and (6.37) we obtain

$$
\begin{aligned}
& \liminf _{h \rightarrow+\infty}-t \int_{\Omega} a_{h}\left(x, \nabla u_{h}\right) \nabla \Phi \phi \mathrm{d} x+t \int_{\Omega} \Phi \phi \mathrm{d} \mu+\int_{\Omega} T_{j}\left(u-T_{i}(u)-t \Phi\right) \phi \mathrm{d} \mu \\
& \quad+\int_{\Omega} \sigma \nabla \phi\left(-t \Phi-T_{j}\left(u-T_{i}(u)-t \Phi\right)\right) \mathrm{d} x \\
& \geqslant \int_{\Omega} a\left(x, \nabla\left(T_{i}(u)+t \Phi\right)\right) \nabla T_{j}\left(u-T_{i}(u)-t \Phi\right) \phi \mathrm{d} x,
\end{aligned}
$$


which gives, letting $i \rightarrow+\infty$

$$
\liminf _{h \rightarrow+\infty}-t \int_{\Omega} a_{h}\left(x, \nabla u_{h}\right) \nabla \Phi \phi \mathrm{d} x \geqslant-t \int_{\Omega} a(x, \nabla(u+t \Phi)) \nabla \Phi \phi \mathrm{d} x .
$$

Finally, dividing by $|t|$ and passing to the limit with respect to $t \rightarrow 0$, we obtain (6.32).

Combining (6.32) and (6.31), we have

$$
\int_{\Omega}(\sigma-a(x, \nabla u)) \nabla \Phi \phi \mathrm{d} x=0,
$$

for every $\Phi \in W_{0}^{1, q^{\prime}}(\Omega)$, with $q<\frac{N}{N-1}$, and for every $\phi \in C^{1}(\bar{\Omega})$.

Let $\xi \in \mathbb{R}^{N}$, with $\xi \neq 0$, and let $\zeta \in C_{c}^{\infty}(\Omega)$; then the choice of $\Phi(x)=\xi x \zeta(x)$ in (6.38) is admissible, and gives

$$
\int_{\Omega}(\sigma-a(x, \nabla u)) \xi \zeta \phi \mathrm{d} x=0
$$

since $\xi x \phi(x) \in C^{1}(\bar{\Omega})$. Now we let $\zeta$ tend to 1 , obtaining

$$
\int_{\Omega}(\sigma-a(x, \nabla u)) \xi \phi \mathrm{d} x=0
$$

for every $\phi \in C^{1}(\bar{\Omega})$, and, finally, $(\sigma(x)-a(x, \nabla u(x))) \xi=0$, for every $\xi \in \mathbb{R}^{N}$ and for almost every $x \in \Omega$, so that (6.5) is proved.

Step 4. We will prove the lower semicontinuity of the "energy", that is

$$
\int_{\Omega} a(x, \nabla u) \nabla T_{j}(u) \mathrm{d} x \leqslant \liminf _{h \rightarrow+\infty} \int_{\Omega} a_{h}\left(x, \nabla u_{h}\right) \nabla T_{j}\left(u_{h}\right) \mathrm{d} x,
$$

for every $j>0$.

Proof of Step 4. To prove (6.39) we need an approximation result for the $G$-convergence (see Lemma 2.3 of [16]).

Lemma 6.4. Let $a_{h}$ be a sequence in $\mathcal{L}\left(c_{0}, c_{1}, \alpha, \beta\right) G$-converging to a function $a$, and let $A_{h}$ and $A$ be the operators associated to $a_{h}$ and a, respectively. Let $v \in W_{0}^{1, p}(\Omega) \cap L^{\infty}(\Omega)$ and $v_{h}$ the solution of (4.1) relative to $A(v)$. Then there exist a decreasing sequence $\varepsilon_{h}$ converging to 0 and a sequence $w_{h} \in W_{0}^{1, p}(\Omega) \cap L^{\infty}(\Omega)$ such that

$$
\begin{aligned}
& w_{h} \rightarrow v \quad \text { weakly in } W_{0}^{1, p}(\Omega), \\
& \left(a_{h}\left(x, \nabla w_{h}\right)-a_{h}\left(x, \nabla v_{h}\right)\right) \rightarrow 0 \quad \text { strongly in } L^{p^{\prime}}(\Omega)^{N}, \\
& \left|w_{h}(x)-v(x)\right| \leqslant \varepsilon_{h} \quad C_{p} \text {-q.e. in } \Omega .
\end{aligned}
$$

Let $v, w_{h} \in W_{0}^{1, p}(\Omega) \cap L^{\infty}(\Omega)$ as in Lemma 6.4. By the monotonicity assumption on $a_{h}(x, \cdot)$ we have, for every $j>0$ :

$$
\int_{\Omega}\left(a_{h}\left(x, \nabla u_{h}\right)-a_{h}\left(x, \nabla w_{h}\right)\right) \nabla T_{j}\left(u_{h}-w_{h}\right) \mathrm{d} x \geqslant 0 .
$$


We use the entropy formulation of $u_{h}$ to obtain

$$
\int_{\Omega} T_{j}\left(u_{h}-w_{h}\right) \mathrm{d} \lambda_{h}+\int_{\Omega} T_{j}\left(u_{h}-w_{h}\right) \mathrm{d} \mu_{h}-\int_{\Omega} a_{h}\left(x, \nabla w_{h}\right) \nabla T_{j}\left(u_{h}-w_{h}\right) \mathrm{d} x \geqslant 0 .
$$

We rewrite the previous inequality as

$$
\left\{\begin{array}{l}
I_{h}+I I_{h}+I I I_{h} \geqslant 0, \\
I_{h}=\int_{\Omega} T_{j}\left(u_{h}-w_{h}\right) \mathrm{d} \lambda_{h}, \\
I I_{h}=\int_{\Omega} T_{j}\left(u_{h}-w_{h}\right) \mathrm{d} \mu_{h}, \\
I I I_{h}=-\int_{\Omega} a_{h}\left(x, \nabla w_{h}\right) \nabla T_{j}\left(u_{h}-w_{h}\right) \mathrm{d} x,
\end{array}\right.
$$

and the term $I I I_{h}$ as

$$
I I I_{h}=\int_{\Omega}\left(a_{h}\left(x, \nabla v_{h}\right)-a_{h}\left(x, \nabla w_{h}\right)\right) \nabla T_{j}\left(u_{h}-w_{h}\right) \mathrm{d} x-\int_{\Omega} a_{h}\left(x, \nabla v_{h}\right) \nabla T_{j}\left(u_{h}-w_{h}\right) \mathrm{d} x .
$$

By (5.3), $T_{j}\left(u_{h}-w_{h}\right)$ is uniformly bounded (with respect to $h$ ) in $W_{0}^{1, p}(\Omega)$, so that $T_{j}\left(u_{h}-w_{h}\right)$ converges weakly in $W_{0}^{1, p}(\Omega)$ to $T_{j}(u-v)$. Thanks to this fact and to (6.41), it is easy to pass to the limit in the first term of $I I I_{h}$. For the second one it is sufficient to use the definition of $v_{h}$ and, again, the weak convergence in $W_{0}^{1, p}(\Omega)$ of $T_{j}\left(u_{h}-w_{h}\right)$, so that

$$
\lim _{h \rightarrow+\infty} I I I_{h}=-\int_{\Omega} a(x, \nabla v) \nabla T_{j}(u-v) \mathrm{d} x .
$$

Analogously we have

$$
\lim _{h \rightarrow+\infty} I I_{h}=\int_{\Omega} T_{j}(u-v) \mathrm{d} \mu,
$$

since we can apply Lemma 5.2. Finally, thanks to (6.42) and by the lipschitzianity of the truncation function, we have:

$$
\liminf _{h \rightarrow+\infty} I_{h}=\liminf _{h \rightarrow+\infty} \int_{\Omega} T_{j}\left(u_{h}-v\right) \mathrm{d} \lambda_{h} .
$$

Combining (6.43), (6.44), and (6.45) we obtain

$$
\liminf _{h \rightarrow+\infty} \int_{\Omega} T_{j}\left(u_{h}-v\right) \mathrm{d} \lambda_{h}+\int_{\Omega} T_{j}(u-v) \mathrm{d} \mu \geqslant \int_{\Omega} a(x, \nabla v) \nabla T_{j}(u-v) \mathrm{d} x,
$$

for every $v \in W_{0}^{1, p}(\Omega) \cap \mathrm{L}^{\infty}(\Omega)$.

Let $t \in(0,1)$; for $i>0$, we use $v=t T_{i}(u)$ as function test in (6.46). Since $t T_{i}(u) \geqslant t T_{i}(\psi) C_{p}$-q.e. in $\Omega$ and since $K_{t T_{i}\left(\psi_{h}\right)}$ converges to $K_{t T_{i}(\psi)}$ in the sense of Mosco (see Remark 4.5), there exist $k \in \mathbb{N}$ and a sequence $z_{h}$ converging to $t T_{i}(u)$ strongly in $W_{0}^{1, p}(\Omega)$ such that $z_{h} \in K_{t T_{i}\left(\psi_{h}\right)}$, for every $h \geqslant k$. We consider the function $\Phi_{h}=T_{i}\left(z_{h}\right)-t T_{i}(u)$, which belongs to $W_{0}^{1, p}(\Omega) \cap \mathrm{L}^{\infty}(\Omega)$ and tends to 0 strongly in $W_{0}^{1, p}(\Omega)$; so we can use (6.18) and the lipschitzianity of the truncation function to deduce that

$$
\liminf _{h \rightarrow+\infty} \int_{\Omega} T_{j}\left(u_{h}-t T_{i}(u)\right) \mathrm{d} \lambda_{h}=\liminf _{h \rightarrow+\infty} \int_{\Omega} T_{j}\left(u_{h}-T_{i}\left(z_{h}\right)\right) \mathrm{d} \lambda_{h} .
$$


Moreover, since, for every $h \geqslant k, T_{i}\left(z_{h}\right) \geqslant t T_{i}\left(\psi_{h}\right) C_{p}$-q.e. in $\Omega$, we estimate the right-hand side of (6.47) as

$$
\liminf _{h \rightarrow+\infty} \int_{\Omega} T_{j}\left(u_{h}-T_{i}\left(z_{h}\right)\right) \mathrm{d} \lambda_{h} \leqslant \liminf _{h \rightarrow+\infty} \int_{\Omega} T_{j}\left(u_{h}-t T_{i}\left(\psi_{h}\right)\right) \mathrm{d} \lambda_{h}=\liminf _{h \rightarrow+\infty} \int_{\Omega} T_{j}\left(u_{h}-t T_{i}\left(u_{h}\right)\right) \mathrm{d} \lambda_{h},
$$

where the last equality follows by the complementarity system (3.21). Finally, using the entropy formulation of $u_{h}$, we get

$$
\begin{aligned}
& \liminf _{h \rightarrow+\infty} \int_{\Omega} T_{j}\left(u_{h}-T_{i}\left(z_{h}\right)\right) \mathrm{d} \lambda_{h} \\
& \quad \leqslant \liminf _{h \rightarrow+\infty}\left(\int_{\Omega} a_{h}\left(x, \nabla u_{h}\right) \nabla T_{j}\left(u_{h}-t T_{i}\left(u_{h}\right)\right) \mathrm{d} x-\int_{\Omega} T_{j}\left(u_{h}-t T_{i}\left(u_{h}\right)\right) \mathrm{d} \mu_{h}\right) \\
& \quad=\liminf _{h \rightarrow+\infty} \int_{\Omega} a_{h}\left(x, \nabla u_{h}\right) \nabla T_{j}\left(u_{h}-t T_{i}\left(u_{h}\right)\right) \mathrm{d} x-\int_{\Omega} T_{j}\left(u-t T_{i}(u)\right) \mathrm{d} \mu,
\end{aligned}
$$

since $T_{j}\left(u_{h}-t T_{i}\left(u_{h}\right)\right)$ converges to $T_{j}\left(u-t T_{i}(u)\right)$ weakly in $W_{0}^{1, p}(\Omega)$ and we can apply Lemma 5.2.

Hence, using in (6.46) $v=t T_{i}(u)$ and combining (6.47) and (6.48), we obtain

$$
\liminf _{h \rightarrow+\infty} \int_{\Omega} a_{h}\left(x, \nabla u_{h}\right) \nabla T_{j}\left(u_{h}-t T_{i}\left(u_{h}\right)\right) \mathrm{d} x \geqslant \int_{\Omega} a\left(x, t \nabla T_{i}(u)\right) \nabla T_{j}\left(u-t T_{i}(u)\right) \mathrm{d} x .
$$

We denote $\int_{\Omega} a_{h}\left(x, \nabla u_{h}\right) \nabla T_{j}\left(u_{h}-t T_{i}\left(u_{h}\right)\right) \mathrm{d} x$ by $J_{h}$, and we split $\Omega$ into the sets where $\left|u_{h}\right| \leqslant i$ and where $\left|u_{h}\right|>i$, so that

$$
J_{h}=\int_{\left\{\left|u_{h}\right| \leqslant i\right\}} a_{h}\left(x, \nabla u_{h}\right) \nabla T_{j}\left((1-t)\left(u_{h}\right)\right) \mathrm{d} x+\int_{\left\{\left|u_{h}\right|>i\right\} \cap\left\{\left|u_{h}-t T_{i}\left(u_{h}\right)\right| \leqslant j\right\}} a_{h}\left(x, \nabla u_{h}\right) \nabla u_{h} \mathrm{~d} x .
$$

Observing that $\left\{\left|u_{h}-t T_{i}\left(u_{h}\right)\right| \leqslant j\right\} \subseteq\left\{\left|u_{h}\right| \leqslant j+t i\right\}$, if we choose $j<(1-t) i$, we have that $\left\{\left|u_{h}\right|>i\right\} \cap\left\{\mid u_{h}-\right.$ $\left.t T_{i}\left(u_{h}\right) \mid \leqslant j\right\}$ is empty, and

$$
J_{h}=\int_{\left\{\left|u_{h}\right| \leqslant i\right\}} a_{h}\left(x, \nabla u_{h}\right) \nabla T_{j}\left((1-t)\left(u_{h}\right)\right) \mathrm{d} x \leqslant \int_{\Omega} a_{h}\left(x, \nabla u_{h}\right) \nabla T_{j}\left((1-t)\left(u_{h}\right)\right) \mathrm{d} x,
$$

since the integrand is nonnegative. Analogously

$$
\int_{\Omega} a\left(x, t \nabla T_{i}(u)\right) \nabla T_{j}\left(u-t T_{i}(u)\right) \mathrm{d} x=\int_{\{|u| \leqslant i\}} a\left(x, t \nabla T_{i}(u)\right) \nabla T_{j}((1-t) u) \mathrm{d} x,
$$

so that (6.49) becomes

$$
\liminf _{h \rightarrow+\infty} \int_{\Omega} a_{h}\left(x, \nabla u_{h}\right) \nabla T_{j}\left((1-t)\left(u_{h}\right)\right) \mathrm{d} x \geqslant \int_{\{|u| \leqslant i\}} a\left(x, t \nabla T_{i}(u)\right) \nabla T_{j}((1-t) u) \mathrm{d} x .
$$

Letting $i$ tend to $+\infty$, we rewrite the previous inequality as

$$
\liminf _{h \rightarrow+\infty} \int_{\Omega} a_{h}\left(x, \nabla u_{h}\right) \nabla T_{j}\left((1-t)\left(u_{h}\right)\right) \mathrm{d} x \geqslant \int_{\Omega} a(x, t \nabla u) \nabla T_{j}((1-t) u) \mathrm{d} x
$$

or, equivalently,

$$
(1-t) \liminf _{h \rightarrow+\infty} \int_{\left\{(1-t)\left|u_{h}\right| \leqslant j\right\}} a_{h}\left(x, \nabla u_{h}\right) \nabla u_{h} \mathrm{~d} x \geqslant(1-t) \int_{\{(1-t)|u| \leqslant j\}} a(x, t \nabla u) \nabla u \mathrm{~d} x
$$


for every $j>0$. Let $n>0$ and $j=(1-t) n$; then we can rewrite (6.50) as

$$
\liminf _{h \rightarrow+\infty} \int_{\Omega} a_{h}\left(x, \nabla u_{h}\right) \nabla T_{n}\left(u_{h}\right) \mathrm{d} x \geqslant \int_{\Omega} a(x, t \nabla u) \nabla T_{n}(u) \mathrm{d} x .
$$

Finally, letting $t$ tend to $1^{-}$, we obtain (6.39).

Step 5. We will prove (6.6).

Proof of Step 5. The proof is quite similar to that of Step 2, so we will often refer to it.

Let $t>0$; then, for every $k>0$, we have that $t T_{k}(u) \geqslant t T_{k}(\psi) C_{p}$-q.e. in $\Omega$. Since $K_{t} T_{k}\left(\psi_{h}\right)$ converges to $K_{t T_{k}(\psi)}$ in the sense of Mosco (see Remark 4.5), there exist $n \in \mathbb{N}$ and a sequence $\Phi_{h}$ converging to $t T_{k}(u)$ strongly in $W_{0}^{1, p}(\Omega)$ such that $\Phi_{h} \in K_{t} T_{k}\left(\psi_{h}\right)$ for every $h \geqslant n$. For $i>0$ we consider the solution $v_{h}$ of $V I\left(A_{h}, A\left(T_{i}(u)+t T_{k}(u)\right), \psi_{h}+\Phi_{h}\right)$ and the obstacle reaction $\eta_{h}$ associated with it; as in the proof of Step 2, we deduce by Theorem 3.1 of [16] that

$$
\begin{aligned}
& v_{h} \rightarrow T_{i}(u)+t T_{k}(u) \quad \text { weakly in } W_{0}^{1, p}(\Omega), \\
& \eta_{h} \rightarrow 0 \quad \text { weakly in } W^{-1, p^{\prime}}(\Omega),
\end{aligned}
$$

since $T_{i}(u)+t T_{k}(u)$ is the solution of $\operatorname{VI}\left(A, A\left(T_{i}(u)+t T_{k}(u)\right), \psi+t T_{k}(u)\right)$. We have also, by (6.24), that

$$
\begin{gathered}
\liminf _{h \rightarrow+\infty} \int_{\Omega} T_{j}\left(u_{h}-v_{h}\right) \mathrm{d} \lambda_{h}+\int_{\Omega} T_{j}\left(u-T_{i}(u)-t T_{k}(u)\right) \mathrm{d} \mu \\
\geqslant \int_{\Omega} a\left(x, \nabla\left(T_{i}(u)+t T_{k}(u)\right)\right) \nabla T_{j}\left(u-T_{i}(u)-t T_{k}(u)\right) \mathrm{d} x .
\end{gathered}
$$

On the other hand, by (6.26), we have that

$$
\begin{aligned}
\liminf _{h \rightarrow+\infty} \int_{\Omega} T_{j}\left(u_{h}-v_{h}\right) \mathrm{d} \lambda_{h} & \leqslant \liminf _{h \rightarrow+\infty} \int_{\Omega} T_{j}\left(-\Phi_{h}\right) \mathrm{d} \lambda_{h} \leqslant \liminf _{h \rightarrow+\infty} \int_{\Omega} T_{j}\left(-t T_{k}\left(\psi_{h}\right)\right) \mathrm{d} \lambda_{h} \\
& =\liminf _{h \rightarrow+\infty} \int_{\Omega} T_{j}\left(-t T_{k}\left(u_{h}\right)\right) \mathrm{d} \lambda_{h},
\end{aligned}
$$

where the last inequalities follow, on one hand, by the fact that $\Phi_{h} \in K_{t T_{k}\left(\psi_{h}\right)}$, for $h$ large enough, on the other, by the complementarity system (3.21). Thanks to (6.52) we rewrite (6.51) as

$$
\begin{gathered}
\liminf _{h \rightarrow+\infty} \int_{\Omega} T_{j}\left(-t T_{k}\left(u_{h}\right)\right) \mathrm{d} \lambda_{h}+\int_{\Omega} T_{j}\left(u-T_{i}(u)-t T_{k}(u)\right) \mathrm{d} \mu \\
\geqslant \int_{\Omega} a\left(x, \nabla\left(T_{i}(u)+t T_{k}(u)\right)\right) \nabla T_{j}\left(u-T_{i}(u)-t T_{k}(u)\right) \mathrm{d} x .
\end{gathered}
$$

Let us choose $j \geqslant t k$ and $i>k$; if we split the integral in the right-hand side of (6.53) into the sets where $|u| \leqslant i$ and where $|u|>i$ we obtain by (2.3):

$$
-t \int_{\{|u| \leqslant i\}} a\left(x, \nabla\left(u+t T_{k}(u)\right)\right) \nabla T_{k}(u) \mathrm{d} x=-t \int_{\Omega} a\left(x, \nabla\left(T_{k}(u)(1+t)\right)\right) \nabla T_{k}(u) \mathrm{d} x .
$$

As in the proof of Step 2, we let $i$ tend to $+\infty$ in (6.53), so that, using (6.54), we easily get

$$
-t \limsup _{h \rightarrow+\infty} \int_{\Omega} T_{k}\left(u_{h}\right) \mathrm{d} \lambda_{h}-t \int_{\Omega} T_{k}(u) \mathrm{d} \mu \geqslant-t \int_{\Omega} a\left(x, \nabla\left(T_{k}(u)(1+t)\right)\right) \nabla T_{k}(u) \mathrm{d} x,
$$


or, equivalently, using the entropy formulation of $u_{h}$

$$
\begin{aligned}
& -t \limsup _{h \rightarrow+\infty}\left(\int_{\Omega} a_{h}\left(x, \nabla u_{h}\right) \nabla T_{k}\left(u_{h}\right) \mathrm{d} x-\int_{\Omega} T_{k}\left(u_{h}\right) \mathrm{d} \mu_{h}\right)-t \int_{\Omega} T_{k}(u) \mathrm{d} \mu \\
& \geqslant-t \int_{\Omega} a\left(x, \nabla\left(T_{k}(u)(1+t)\right)\right) \nabla T_{k}(u) \mathrm{d} x,
\end{aligned}
$$

for every $k>0$. On the other hand, Lemma 5.2 implies that

$$
\lim _{h \rightarrow+\infty} \int_{\Omega} T_{k}\left(u_{h}\right) \mathrm{d} \mu_{h}=\int_{\Omega} T_{k}(u) \mathrm{d} \mu,
$$

so that (6.55) becomes

$$
-t \limsup _{h \rightarrow+\infty} \int_{\Omega} a_{h}\left(x, \nabla u_{h}\right) \nabla T_{k}\left(u_{h}\right) \mathrm{d} x \geqslant-t \int_{\Omega} a\left(x, \nabla\left(T_{k}(u)(1+t)\right)\right) \nabla T_{k}(u) \mathrm{d} x .
$$

Finally, dividing by $t$ and passing to the limit as $t \rightarrow 0^{+}$, we have, for every $k>0$

$$
\limsup _{h \rightarrow+\infty} \int_{\Omega} a_{h}\left(x, \nabla u_{h}\right) \nabla T_{k}\left(u_{h}\right) \mathrm{d} x \leqslant \int_{\Omega} a(x, \nabla u) \nabla T_{k}(u) \mathrm{d} x,
$$

which, combined with (6.39) gives (6.6).

Remark 6.5. If we choose in Theorem 6.1 as obstacles $\psi_{h}=\psi=-\infty$, we recover Theorem 3.2 of [4], which concerns the continuous dependence of the entropy solutions under perturbations of the operator $A$.

Remark 6.6. Let us remark that we cannot prove Theorem 6.1 under the assumption that $\mu_{h}$ converges to $\mu$ in the *-weak topology of $\mathcal{M}_{b}(\Omega)$ (see Example 4.5 of [13]).

Corollary 6.7. Let a be in $\mathcal{L}\left(c_{0}, c_{1}, \alpha, \beta\right)$ and $A$ be the operator associated with it. Let us assume (6.1), (6.2) (with $A_{h}=A$, for every $h>0$ ), and (6.3), with $K_{\psi_{h}}$ converging to $K_{\psi}$ in the sense of Mosco. Finally, consider $\mu_{h}$, $\mu \in \mathcal{M}_{b, 0}^{p}(\Omega)$, with $\mu_{h}$ converging to $\mu$ weakly in $\mathcal{M}_{b}(\Omega)$. Then the solutions $u_{h}$ and $u$ of the obstacle problems $O P\left(A, \mu_{h}, \psi_{h}\right)$ and $\operatorname{OP}(A, \mu, \psi)$, respectively, satisfy

$$
T_{j}\left(u_{h}\right) \rightarrow T_{j}(u) \quad \text { strongly in } W_{0}^{1, p}(\Omega), \text { for every } j>0 .
$$

Proof. By Theorem 6.1 (with $a_{h}=a$, for every $h>0$ ) we have that $T_{j}\left(u_{h}\right)$ converges to $T_{j}(u)$ weakly in $W_{0}^{1, p}(\Omega)$, and

$$
\int_{\Omega} a\left(x, \nabla u_{h}\right) \nabla T_{j}\left(u_{h}\right) \mathrm{d} x \rightarrow \int_{\Omega} a(x, \nabla u) \nabla T_{j}(u) \mathrm{d} x, \quad \text { for every } j>0 .
$$

On the other hand, if the function $a$ is fixed, working as in the proof of Theorem 6.1 of [3], it can be proved that $\nabla u_{h}$ converges to $\nabla u$ almost everywhere in $\Omega$. Since $a(x, \cdot)$ is a Carathéodory function, also $a\left(x, \nabla T_{j}\left(u_{h}\right)\right)$ tends to $a\left(x, \nabla T_{j}(u)\right)$ almost everywhere in $\Omega$.

Moreover, thanks to $(2.1), a\left(x, \nabla T_{j}\left(u_{h}\right)\right)$ is uniformly (with respect to $h$ ) bounded in $L^{p^{\prime}}(\Omega)^{N}$; so we deduce that

$$
a\left(x, \nabla T_{j}\left(u_{h}\right)\right) \rightarrow a\left(x, \nabla T_{j}(u)\right) \quad \text { weakly in } L^{p^{\prime}}(\Omega)^{N} .
$$


Combining (6.57) and (6.58), we have:

$$
\lim _{h \rightarrow+\infty} \int_{\Omega}\left(a\left(x, \nabla T_{j}\left(u_{h}\right)\right)-a\left(x, \nabla T_{j}(u)\right)\right) \nabla\left(T_{j}\left(u_{h}\right)-T_{j}(u)\right) \mathrm{d} x=0,
$$

which implies that $T_{j}\left(u_{h}\right)$ converges to $T_{j}(u)$ strongly in $W_{0}^{1, p}(\Omega)$.

\section{References}

[1] H. Attouch, C. Picard, Problemes variationnels et theorie du potential non lineaire, Ann. Faculté Sci. Toùlouse 1 (1979) 89-136.

[2] J.J. Benedetto, Real Variable and Integration, Teubner, Stuttgart, 1976.

[3] L. Benilan, L. Boccardo, T. Galloüet, R. Gariepy, M. Pierre, J.L. Vazquez, An $L^{1}$ theory of existence and uniqueness of nonlinear elliptic equations, Ann. Scuola Norm. Sup. Pisa 22 (1995) 240-273.

[4] L. Boccardo, Homogenization and continuous dependence for Dirichlet problems in $L^{1}$, in: Partial Differential Equation Methods in Control and Shape Analysis, in: Lecture Notes in Pure and Appl. Math., vol. 188, Dekker, New York, 1997, pp. 41-52.

[5] L. Boccardo, G.R. Cirmi, Existence and uniqueness of solutions of unilateral problems with $L^{1}$-data, Manuscript.

[6] L. Boccardo, T. Galloüet, Problèmes unilatéraux avec données dans $L^{1}$, C. R. Acad. Sci. Paris Sér. I 311 (1990) 617-619.

[7] L. Boccardo, T. Galloüet, L. Orsina, Existence and uniqueness of entropy solutions for nonlinear elliptic equations with measure data, Ann. Inst. H. Poincaré 13 (1996) 539-551.

[8] L. Boccardo, F. Murat, Homogenization of nonlinear unilateral problems, in: Composite media and homogenization theory, in: Progr. Nonlinear Differential Equations Appl., vol. 5, Birkhäuser Boston, Boston, 1991, pp. 81-105.

[9] L. Boccardo, F. Murat, Nouveaux résultats de convergence dans des problèmes unilateraux, in: Nonlinear Partial Differential Equations and their Applications (Collège de France seminar), in: Pitman Res. Notes Math., vol. 60, 1962, pp. 64-85.

[10] J. Casado Diaz, G. Dal Maso, A weak notion of convergence in capacity with applications to thin obstacle problems, in: Calculus of Variation and Related Topics (Proceedings Haifa, Israel), in: Pitman Res. Notes Math. Ser., 1999.

[11] V. Chiadò Piat, A. Defranceschi, Asymptotic behaviour of quasi-linear problems with Neumann boundary conditions on perforated domains, Appl. Anal. 36 (1990) 65-87.

[12] R. Cirmi, Convergence of the solutions of nonlinear obstacle problems with $L^{1}$-data, Manuscript, 1999.

[13] P. Dall'Aglio, C. Leone, Obstacle problems with measure data and linear operators, Potential Anal. 17 (2002) $45-64$.

[14] G. Dal Maso, On the integral representation of certain local functionals, Ricerche Mat. 32 (1983) 85-113.

[15] G. Dal Maso, Some necessary and sufficient conditions for the convergence of sequences of unilateral convex sets, J. Funct. Anal. 62 (1985) 119-159.

[16] G. Dal Maso, A. Defranceschi, Convergence of unilateral problems for monotone operators, J. Anal. Mat. 53 (1989) $269-289$.

[17] T. Del Vecchio, On the homogenization of a class of pseudomonotone operators in divergence form, Boll. Un. Mat. Ital. 7 (1991) $369-388$.

[18] D. Kinderlehrer, G. Stampacchia, An Introduction to Variational Inequalities and their Applications, Academic Press, New York, 1980.

[19] C. Leone, Existence and uniqueness of solutions for nonlinear obstacle problems with measure data, Nonlinear Anal. 43 (2000) 199-215.

[20] C. Leone, On a class of nonlinear obstacle problems with measure data, Commun. Partial Differential Equations 25 (2000) $2259-2286$.

[21] C. Leone, A. Porretta, Entropy solutions for nonlinear elliptic equations in $L^{1}$, Nonlinear Anal. 32 (1998) 325-334.

[22] J.L. Lions, Quelques méthodes de résolution des problèmes aux limites non linéaires, Dunod, Gauthier-Villars, Paris, 1969.

[23] U. Mosco, Convergence of convex sets and of solutions of variational inequalities, Adv. in Math. 3 (1969) 510-585.

[24] L. Tartar, Cours Peccot au Collège de France, Paris 1977. 